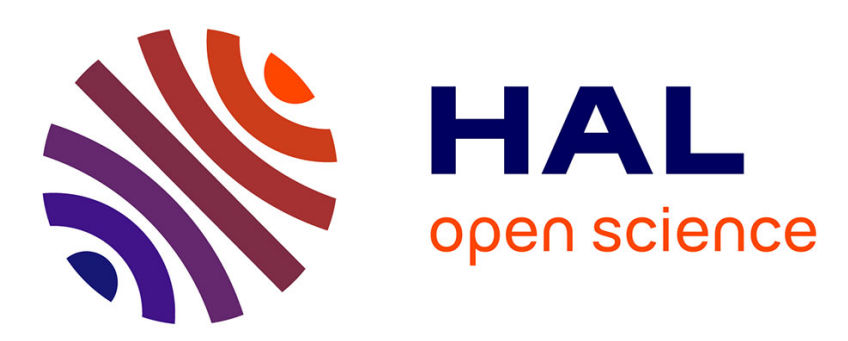

\title{
Les blocs cyclopéens de l'île de Banneg (archipel de Molène, Finistère) : accumulations supratidales de forte énergie
}

\author{
Bernard Fichaut, Serge S. Suanez
}

\section{- To cite this version:}

Bernard Fichaut, Serge S. Suanez. Les blocs cyclopéens de l'île de Banneg (archipel de Molène, Finistère) : accumulations supratidales de forte énergie. Géomorphologie: relief, processus, environnement, 2008, 14 (1), pp.15-32. 10.4000/geomorphologie.5793 . hal-00274142

\section{HAL Id: hal-00274142 \\ https://hal.science/hal-00274142}

Submitted on 8 Aug 2014

HAL is a multi-disciplinary open access archive for the deposit and dissemination of scientific research documents, whether they are published or not. The documents may come from teaching and research institutions in France or abroad, or from public or private research centers.
L'archive ouverte pluridisciplinaire HAL, est destinée au dépôt et à la diffusion de documents scientifiques de niveau recherche, publiés ou non, émanant des établissements d'enseignement et de recherche français ou étrangers, des laboratoires publics ou privés. 

processus, environnement

$1 / 2008$ (2008)

Varia

Bernard Fichaut et Serge Suanez

\title{
Les blocs cyclopéens de l'île de Banneg (archipel de Molène, Finistère) : accumulations supratidales de forte énergie
}

\begin{abstract}
Avertissement
Le contenu de ce site relève de la législation française sur la propriété intellectuelle et est la propriété exclusive de l'éditeur.

Les œuvres figurant sur ce site peuvent être consultées et reproduites sur un support papier ou numérique sous réserve qu'elles soient strictement réservées à un usage soit personnel, soit scientifique ou pédagogique excluant toute exploitation commerciale. La reproduction devra obligatoirement mentionner l'éditeur, le nom de la revue, l'auteur et la référence du document.

Toute autre reproduction est interdite sauf accord préalable de l'éditeur, en dehors des cas prévus par la législation en vigueur en France.
\end{abstract}

\section{revues.org}

Revues.org est un portail de revues en sciences humaines et sociales développé par le Cléo, Centre pour l'édition électronique ouverte (CNRS, EHESS, UP, UAPV).

Référence électronique

Bernard Fichaut et Serge Suanez, « Les blocs cyclopéens de l'île de Banneg (archipel de Molène, Finistère) :

accumulations supratidales de forte énergie », Géomorphologie : relief, processus, environnement

[En ligne], 1/2008 | 2008, mis en ligne le 01 avril 2010, consulté le 12 octobre 2012. URL : http://

geomorphologie.revues.org/5793; DOI : 10.4000/geomorphologie.5793

Éditeur : Groupe français de géomorphologie

http://geomorphologie.revues.org

http://www.revues.org

Document accessible en ligne sur : http://geomorphologie.revues.org/5793

Ce document est le fac-similé de l'édition papier.

(c) Groupe français de géomorphologie 


\title{
Les blocs cyclopéens de l'île de Banneg (archipel de Molène, Finistère) : accumulations supratidales de forte énergie Large clast deposits on Banneg Island (Molène archipelago, Finistère, Brittany): high energy supratidal accumulation
}

\author{
Bernard Fichaut* et Serge Suanez*
}

\begin{abstract}
Résumé
Cette étude porte sur l'analyse morphosédimentaire des blocs cyclopéens de l'île de Banneg, située à la limite nord-ouest de l'archipel de Molène (Finistère). Ces dépôts grossiers sont constitués de dalles rocheuses arrachées au substratum par les houles de tempêtes océaniques attaquant de plein fouet la façade occidentale de l'île. Ils prennent le plus souvent l'allure de remparts culminant entre 7 et 14,5 m d'altitude. Cinquante-deux amas de blocs ont été recensés du nord au sud de l'île, représentant un volume total d'environ $1000 \mathrm{~m}^{3}$. La taille médiane des blocs avoisine $0,8 \times 0,6 \times 0,4 \mathrm{~m}$ pour un poids équivalant à 0,6 tonne tandis que le plus gros bloc, situé au centre de l'île et pesant 32 tonnes, a été projeté à 14 mètres en retrait de la falaise et à une altitude de $9 \mathrm{~m}$. Si la taille des blocs diminue avec la distance au rivage, aucune relation claire n'est notée entre cette dernière et le tri granulométrique. L'étude des conditions hydrodynamiques responsables de la mise en place de ces dépôts a été effectuée à partir des données météo-marines enregistrées durant les tempêtes de l'hiver 1989-1990. Elle montre que les niveaux d'eau extrêmes de débordement ont pu atteindre près de $19 \mathrm{~m}$ de hauteur, soit 5 à $10 \mathrm{~m}$ au dessus des falaises. Toutefois, la submersion a été beaucoup plus importante et fréquente au centre et au sud qu'au nord et au centre nord de l'île. Un rétrocalcul effectué au cours de la période 1979-2002 indique qu'aucun épisode aussi morphogène que ceux de l'hiver 19891990 n'a été enregistré durant ces vingt-quatre années. Il apparaît toutefois que la décennie 1979-1990 a été marquée par un nombre important d'épisodes morphogènes, alors que la fréquence des tempêtes diminue nettement durant la suivante, de 1990 à 2002.
\end{abstract}

Mots clés : blocs cyclopéens, île, run-up, tempête.

\begin{abstract}
This study is based on a morphosedimentary analysis of cyclopean blocks on the coasts of Banneg Island, which is located at the northwestern extremity of the Molène archipelago in Finistère. These deposits correspond to tabular clasts detached from the substratum by giant oceanic storm waves directly impacting the western shoreline of the island. An analysis of these clasts based on DGPS topographic surveys was carried out. Most of them correspond to imbricated clasts forming ridges that reach the highest elevation between 7 and $14.5 \mathrm{~m}$. Fifty-two imbricated clasts on the island were inventoried from the north to south, representing a total volume of $1000 \mathrm{~m}^{3}$. The three median dimensions of clasts are $0.8 \times 0.6 \times 0.4 \mathrm{~m}$ while the median weight is $0.6 \mathrm{t}$. The largest imbricated clast unit, No. 28, measuring $5.3 \times 3.9 \times 0.5 \mathrm{~m}$ and weighing approximately $32 \mathrm{t}$, is located in the centre of the island: it has been deposited at $9 \mathrm{~m}$ in elevation and $14 \mathrm{~m}$ inland from the edge of the cliff. Sediment analysis shows that clast size decreases with distance from the shoreline but there is no relationship between sorting and distance inland. A study of hydrodynamic conditions responsible for clast transport was undertaken by observing the 1989-1990 winter storms. This analysis shows that extreme water levels causing submersion may have reached heights of approximately $19 \mathrm{~m}$, i.e. 5 to $10 \mathrm{~m}$ higher than the tops of existing sea-cliffs. At the same time, submersion processes were more powerful and frequent in the centre and south of the island. A back-calculation over the 1979-2002 time interval shows that no event as powerful as those of the 1989-1990 winter was recorded during the last twenty-four years. Nevertheless, it appears that whereas the 1979-1990 decade was characterised by important morphogenetic events, the following one (1990-2002) experienced a sharp decrease in storm events.
\end{abstract}

Key words: clasts (cyclopean blocks), island, run-up, storm.

\section{Abridged English version}

This study is based on a morphosedimentary analysis of clasts (cyclopean blocks) on the coast of Banneg Island located at the north-western limit of the archipelago of Molène in Finistère (fig. 1). This archipelago consists of nineteen small islands and reefs covering a total surface area of 195 ha and represents the emerged part of a large

* GEOMER LETG UMR 6554 CNRS, Institut Universitaire Européen de la Mer, Place Nicolas Copernic, 29280 Plouzané. Courriel :

bernard.fichaut@univ-brest.fr ; serge.suanez@univ-brest.fr 
continental platform of about 15,000 ha extending at depths between 0 and $10 \mathrm{~m}$. Banneg Island differs from the other, more sheltered islands because it is located on the edge of the deep Fromveur channel, which separates the archipelago from Ouessant Island. The eastern sheltered coast presents gentle slopes but no cliffs. On the contrary, the exposed western coast is formed by steep cliffs up to 10 to $14 \mathrm{~m}$ high (fig. 2). In this area the retreat of the coast is associated with tabular clast transports by overturning. These deposits form imbricated and/or trains of imbricated clasts. These high-energy sedimentation processes are related to extreme western and north-western waves $\left(270^{\circ}\right.$ to $310^{\circ}$ ), which represent $60 \%$ of the annual regime. Southwestern waves are also well represented but in a lesser proportion (20\% of the annual regime). The most frequent significant wave height reaches about $1.5 \mathrm{~m}$ and represents $30 \%$ of the annual regime (fig. 3). The most frequent maximum heights, which represent 25 to $30 \%$ of the annual regime, reach $2.5 \mathrm{~m}$ but may exceed 16 to $18 \mathrm{~m}$ (fig. 3A). The most frequent significant periods are between 8 and 9 seconds and represent $30 \%$ of the annual regime (fig. 3B). The most frequent maximum periods reach 13.5 seconds but they may reach 24 seconds (fig. 3B).

The morphological study of imbricated clasts was based on DGPS topographic surveys carried out between October 2005 and June 2006. Fifty-two imbricated clasts were surveyed from the north to south, representing a total volume of $1000 \mathrm{~m}^{3}$ (fig. 4C). These imbricated clasts were divided into six groups mainly located ahead of coves separated by headlands (fig. 4A). The clasts correspond to ridges forming an arc-like pattern in the south (fig. 5A) or forming an elongated train parallel to the coast such as near the centre where they also are biggest (fig. 5B). In some parts, deposits also correspond to isolated or individual clasts. It is also in the central area that their arrangement is most remarkable owing to the fact that they locally form triple ridges parallel to each other and distinctly separated by bedrock or turf.

The analysis of deposits accumulated in the six focus areas shows that the volume of clasts is not correlated with either the height or slope of the existing cliff (fig. 6A and 6B). There is also no correlation between deposit distance inland and cliff height or slope (fig. 6C and 6D). From north to south, individual clast units can be distinguished by their own morphodynamic signatures, which are mainly related to the morphology of the tidal zone and the shallow submarine topography seaward of the cliff. For some of them, the arrangement of imbricated clasts shows a seaward dip that suggests wave directions linked to depositional processes (fig. 5C). Dip measurements testify to the predominant westerly $\left(270^{\circ}\right)$ and northwesterly $\left(330^{\circ}\right)$ waves respectively for the areas of the central and southern areas (fig. 4B).

Measurements of 908 block sizes were carried out. The median size was $0.8 \times 0.6 \times 0.4 \mathrm{~m}$ with a weight of 0.6 tons. Yet, clasts varied considerably in size, with the largest measuring $5.3 \times 3.9 \times 0.5 \mathrm{~m}$ and weighing approximately 32 tons. This corresponds to a large tabular clast located in the centre of the island. It has been deposited $14 \mathrm{~m}$ inland from the edge of the cliff at $9 m$ a.s.l. Sediment analysis shows that the size of clasts becomes smaller with increasing distance from the shoreline (fig. 7A). On the contrary, there is no correlation between sorting and distance inland (fig. $7 B$ and $7 C)$.

The study of hydrodynamic conditions responsible for clast transport was undertaken by observing the 1989-1990 winter storms. Following these events, a field survey was carried out in spring 1990 by Hallégouët and Fichaut showing important morphosedimentary and biological changes (fig. 8). Meteorological and marine data recorded during this period show that three storm events distinctly appear between December 1st, 1989 and February 28th, 1990 (fig. 9). Extreme water levels observed during these events were analyzed while taking into account surge (measured tide) and run-up (waves) parameters. The latter component was calculated using Mase's (1989) formula:

$$
\begin{aligned}
& \frac{R^{T}{ }_{\text {max }}}{H_{\text {sig }}}=2,32 \xi_{o}^{0,77} \\
& \frac{R^{T}{ }_{2 \%}}{H_{\text {sig }}}=1,86 \xi_{o}^{0,71}
\end{aligned}
$$

where $R_{\text {max }}^{T}$ corresponds to the highest run-up; $R^{T}{ }_{2 \%}$ corresponds to the $2 \%$ excess run-up height; $H_{\text {sig }}$ is deep water significant wave height; $C=2.32$ for $R^{T}{ }_{\text {max }}$ and 1.86 for $R^{T}{ }_{2 \%}$; $\xi_{o}$ is d'Iribarren number (Battjes, 1974):

$$
\xi_{o}=\frac{\tan \beta}{\left(H_{\text {sig }} / L_{o}\right)^{1 / 2}}
$$

This analysis shows that extreme water levels causing submersion may have reached approximately $19 \mathrm{~m}$ a.s.l., i.e. 5 to $10 \mathrm{~m}$ higher than the top of the sea-cliff (fig. 10). At the same time, submersion events have been higher and more frequent in the centre and south of the island (fig. 10B). This could explain the large volume of clasts accumulated in these areas. By contrast, submersion has been less frequent in the northern part of the island (fig. 10A), causing minor environmental degradation (fig. 11). Finally, retrocalculation over the 19792002 period was made in order to make an inventory of morphogenic events that could have generated clast transport. Results show that no event as powerful as those of the 1989-1990 winter was recorded during the last twenty-four years (fig. 12). Nevertheless, it appears that the 1979-1990 decade was characterised by several morphogenic events whereas the subsequent decade (1990-2002) has experienced a sharp decrease in storm events. These variations could be attributed to the switch from a negative towards a positive phase in the North Atlantic Oscillation index.

\section{Introduction}

L'origine du terme bloc cyclopéen revient à B. Hallégouët, géomorphologue de l'université de Bretagne Occidentale, qui l'utilisa pour la première fois en 1982 pour un article publié dans la revue Penn ar Bed. Décrivant les dépôts grossiers au sommet de l'île de Banneg, il les qualifia ainsi en référence aux murs cyclopéens constitués d'énormes blocs de plusieurs tonnes entassés les uns sur les autres, que les civi- 
lisations antiques grecques mycéniennes du XVIII $\mathrm{I}^{\mathrm{e}} \mathrm{au}$ XII siècle av. J.C. érigeaient pour protéger leurs royaumes, à l'exemple de la ville fortifiée de Mycènes. Le terme disparut toutefois de l'article originel à la demande d'A. Guilcher qui estimait que la référence à une construction d'origine anthropique ne pouvait s'appliquer à une forme naturelle (Hallégouët, com. pers.). Deux ans après, le terme sera définitivement adopté par la communauté scientifique car on le trouve dans un texte de B. Hallégouët (1984) et un an plus tard, dans une étude portant sur l'archipel des Tuamotu (Bourrouilh-Le Jan et Talandier, 1985). Une traduction anglophone, cyclopean blocks, sera même proposée par P. J. Hearty (1997). L'étude présentée dans cet article se situe donc dans la continuité des travaux antérieurs réalisés sur les blocs cyclopéens de l'île de Banneg (Hallégouët, 1982a, 1984 ; Fichaut et Hallégouët, 1989). Reposant sur de nouvelles techniques de mesure de terrain et de traitement de données météo-marines, elle se propose d'approfondir l'analyse morphosédimentaire de ces dépôts et l'étude des processus dynamiques à l'origine de leur mise en place. très grossiers qui résultent de dynamiques marines de très forte énergie (Dawson, 1999 ; Whelan et Keating, 2004). Dans un premier temps, ils ont été étudiés afin de caractériser et d'interpréter leur présence dans des formations pléistocènes (Moore et Moore, 1984 ; Paskoff, 1991 ; Hearty, 1997 ; Hartley et al., 2001) et holocènes (Dawson, 1999 ; Dawson et Shi, 2000 ; Nakata et Kawana, 1993 ; Atwater, 1992 ; Long et al., 1989 ; Kelletat et Schellmann, 2002). Dans la plupart des cas, ces dépôts grossiers ont alors été
Les blocs cyclopéens appartiennent à la famille des dépôts

décrits comme le résultat de l'action de vagues géantes de tsunamis (Young et al., 1996 ; Bryant et al., 1992 ; Clague et al., 1999 ; Pinegina et Bourgeois, 2001 ; Gianfreda et al., 2001 ; Bondevik et al., 2005). Dans un second temps, les recherches ont privilégié l'analyse des processus sur des périodes beaucoup moins longues, pluridécennales à pluriséculaires, en essayant de faire la part entre l'action des tsunamis et des événements météo-marins extrêmes de type tempêtes, ouragans, hurricanes, typhons, cyclones, etc. (Nott, 1997, 2000, 2003, 2004 ; Bourrouilh-Le Jan et Taladier, 1985 ; Jones et Hunter, 1992 ; Nanayama et al., 2000 ; Goff et al., 2006 ; Noormets et al., 2002 ; Keating et al., 2004).

L'objectif de notre étude a été dans un premier temps de reprendre l'analyse morphosédimentaire des dépôts de l'île de Banneg. Ce travail a reposé sur des levés topo-morphologiques effectués au DGPS qui ont permis de cartographier, d'analyser et de quantifier l'ensemble des amas ; l'analyse morphométrique et granulométrique a été, quant à elle, réalisée sur un échantillon de plus de 900 blocs. Dans un second temps, cette recherche s'est attachée à analyser les modalités de mise en place de ces dépôts. À ce titre, un travail théorique reposant sur le traitement des données de houle acquises entre 1979 et 2002 a été effectué afin de quantifier les effets du run-up qui ont été très souvent décrits comme le moteur de l'arrachement et du déplacement des blocs cyclopéens (Noormets et al., 2004 ; Dawson, 1999 ; Dawson et Shi, 2000).

\section{Contexte géographique et hydrodynamique}

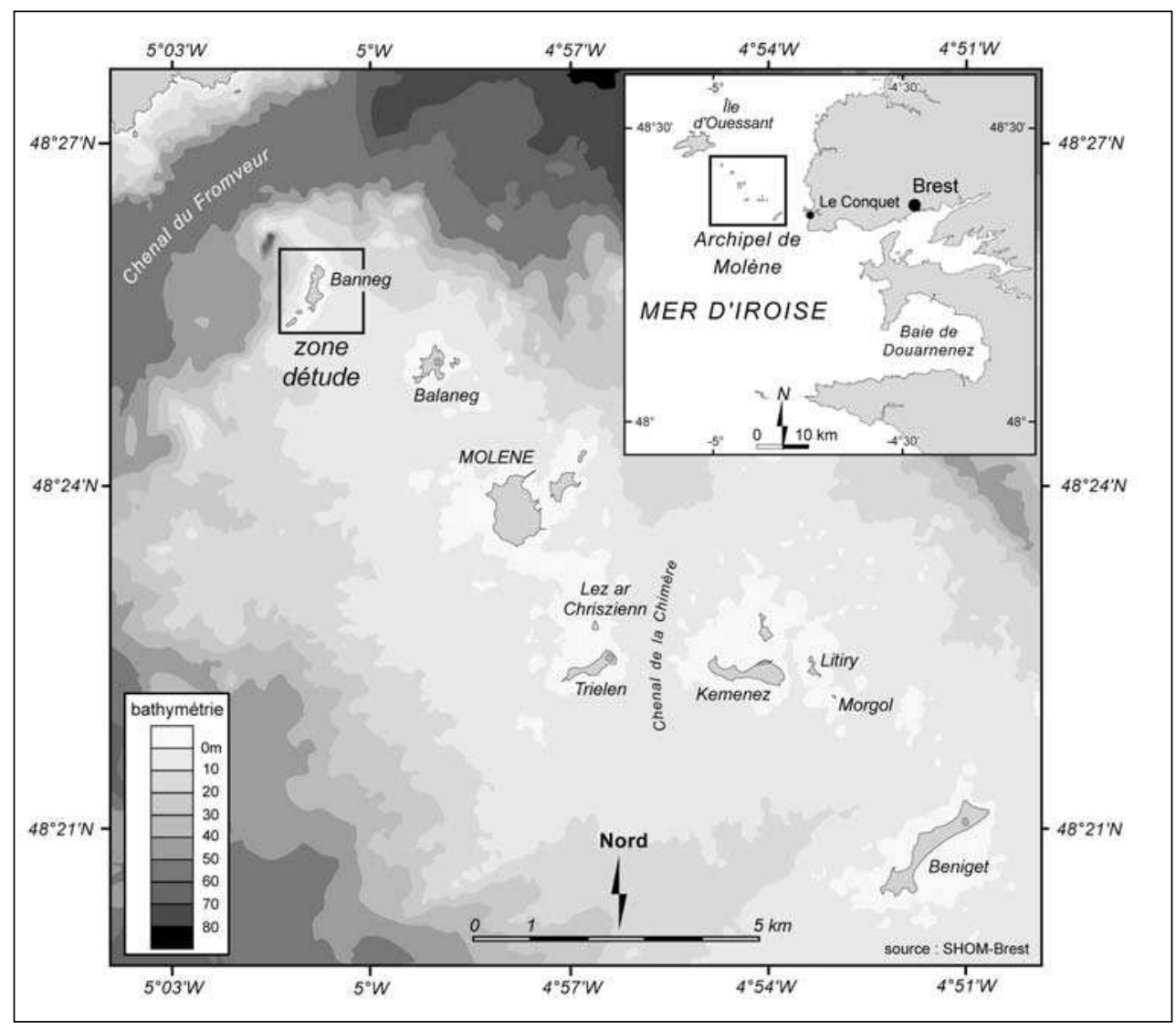

L'île de Banneg se situe dans la Réserve naturelle d'Iroise, dans la partie septentrionale de l'archipel de Molène en mer d'Iroise (fig. 1). Situé au large de la côte nord-ouest de la Bretagne, cet archipel est composé de 19 îles et îlots couvrant une superficie totale de 195 ha et représentant la partie émergée d'un vaste plateau sous-marin qui s'étend sur environ 15000 ha au-dessus de l'isobathe 10 mètres (Guilcher, 1959 ; Hallégouët, 1982b). L'île de Banneg se distingue des autres îlots, relativement abrités, par sa position excentrée au nord-ouest, immédiatement en bordure du

Fig. 1 - Carte de localisation de l'archipel de Molène et de l'île de Banneg.

Fig. 1 - Location map of the Molène archipelago and Banneg island. 
Fig. 2 - Vue aérienne oblique de l'île de Banneg (cliché E. Le Droff, Le Télégramme).

Fig. 2 - Oblique aerial photograph of Banneg Island (photo: E. Le Droff, Le Télégramme).

profond chenal du Fromveur qui sépare l'archipel de l'île d'Ouessant. L'originalité de Banneg réside dans la dissymétrie entre ses côtes est et ouest (fig. 2). Le littoral oriental, abrité et dépourvu de falaises, présente des pentes assez faibles ; ses formes d'accumulation sont constituées de cordons de galets et de petits placages sableux assimilables à des plages. À l'inverse, la côte occidentale exposée à la houle est entaillée en falaises plus ou moins verticales qui cul-

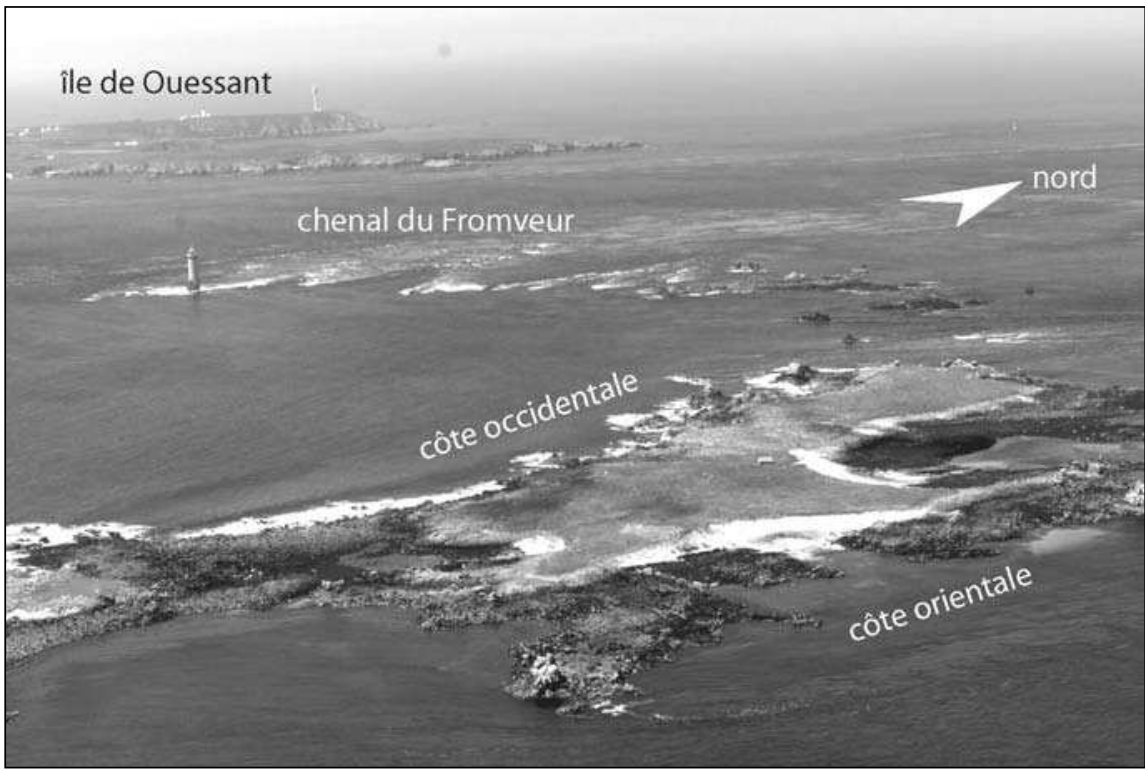
minent entre 10 et $14 \mathrm{~m}$ d'altitude NGF. Contrairement à ce qui se passe pour la plupart des côtes d'abrasion où le recul de la falaise produit une accumulation de matériaux à son pied, l'érosion se manifeste ici par des projections de dalles rocheuses, qui s'accumulent sous la forme d'amas de blocs plus ou moins organisés, au sommet de l'île.

La mer d'Iroise est caractérisée par un régime macrotidal semi-diurne dont le marnage varie entre $3 \mathrm{~m}$ en marée de morte-eau (coefficient 45) et $7 \mathrm{~m}$ en vive-eau exceptionnelle (coefficient 120). Le zéro hydrographique calculé à partir des enregistrements marégraphiques du port du Conquet (marégraphe le plus représentatif de la mer d'Iroise), se situe à 3,48 m sous le zéro topographique IGN 69. L'archipel de Molène est directement ouvert à la houle océanique atlantique. Les mesures effectuées au large de Ouessant par la bouée-phare BEAIII sur la période 1985-2001 montrent que la hauteur significative $\left(\mathrm{H}_{\text {sig }}\right)$ modale des houles est d'environ $1,5 \mathrm{~m}$ (environ $30 \%$ du régime annuel) (fig. 3A). Les hauteurs maximales les plus fréquentes (entre 25 et $30 \%$ du régime annuel) atteignent $2,5 \mathrm{~m}$, mais peuvent toutefois dépasser 16 à $18 \mathrm{~m}$ (fig. 3A). La période significative $\left(\mathrm{T}_{\mathrm{sig}}\right)$ modale est comprise entre 8 et 9 secondes (environ $30 \%$ du régime annuel), tandis que la période maximale modale est égale à 13,5 secondes mais peut atteindre 24 secondes (fig. 3B). Dans une étude récente, ces enregistrements ont été utilisés pour valider, par comparaison, des données de houles calculées au même point par simulation numérique sur la période 1979-2002 (Benoit et al., 2006). Ces simulations sont effectuées avec le logiciel de modélisation des états de mer TOMAWAC (développé par EDF-LNHE avec le soutien du CETMEF) qui utilise comme forçage atmosphérique les champs de vent issus de la ré-analyse ERA-40 du Centre Européen de Prévision Météorologique à Moyen Terme (ECMWF). La comparaison entre les mesures in situ et les simulations numériques a montré que les enregistrements avaient tendance à sous-estimer systématiquement les valeurs de hauteur des plus fortes houles. Ce biais a par ailleurs été confirmé lors de comparaisons avec des mesures effectuées à partir d'une « Datawell » en 2004 et s'explique

par le poids considérable de la bouée-phare BEAIII (80 tonnes) (Benoit et al., 2006). La bouée BEAIII n'étant pas directionnelle, le spectre des directions a été établi à partir de ces simulations numériques (fig. 3C). Il montre une prédominance des houles de secteur ouest à nord-ouest $\left(270^{\circ}\right.$ à $310^{\circ}$ ) qui représentent pratiquement $60 \%$ du régime annuel et sont les plus énergétiques. Les houles de secteur sudouest sont aussi présentes, mais dans une plus faible proportion $\left(20 \%\right.$ du régime annuel) ; leur hauteur $\left(\mathrm{H}_{\mathrm{mo}}\right)$ peut toutefois atteindre $10 \mathrm{~m}$. La figure 3C montre que l'île de Banneg se situe en bordure de la chaussée de Molène marquée par de fortes pentes. Les isobathes 20 et 50 mètres sont respectivement à $250 \mathrm{~m}$ et $2200 \mathrm{~m}$ de la côte occidentale qui reçoit de plein fouet les houles atlantiques dominantes peu réfractées. Les phénomènes d'amortissement à cet endroit sont donc pratiquement inexistants.

\section{Étude morphosédimentaire des amas de blocs cyclopéens}

L'étude morphologique des amas de blocs cyclopéens repose sur des relevés topographiques effectués au DGPS entre 2005 et 2006 . Plus de 15000 points ont été relevés pour restituer le plus finement possible la topographie de l'île et des falaises soumises aux processus d'érosion et de dépôt, ainsi que la position, l'orientation et la forme des amas. Les mesures ont été effectuées en mode RTK (Real Time Kinematics) avec une précision horizontale et verticale respective de $2 \mathrm{~cm}(\mathrm{x}, \mathrm{y})$ et de 1,5 cm (z). Elles ont été raccordées au système géodésique français à partir d'une borne IGN située sur l'île. Le système de géoréférencement retenu ici correspond à celui du Lambert I (Nord de la France), et le traitement des données a été effectué grâce au logiciel Surfer 8.0. La méthode du krigeage avec intégration de lignes de rupture a été retenue comme mode d'interpolation pour la construction des modèles numériques de terrain. Une maille de $0,5 \times 0,5 \mathrm{~m}$ a été choisie afin d'optimiser la pertinence du rendu topographique. À partir du modèle numérique de terrain, une carte en isolignes 


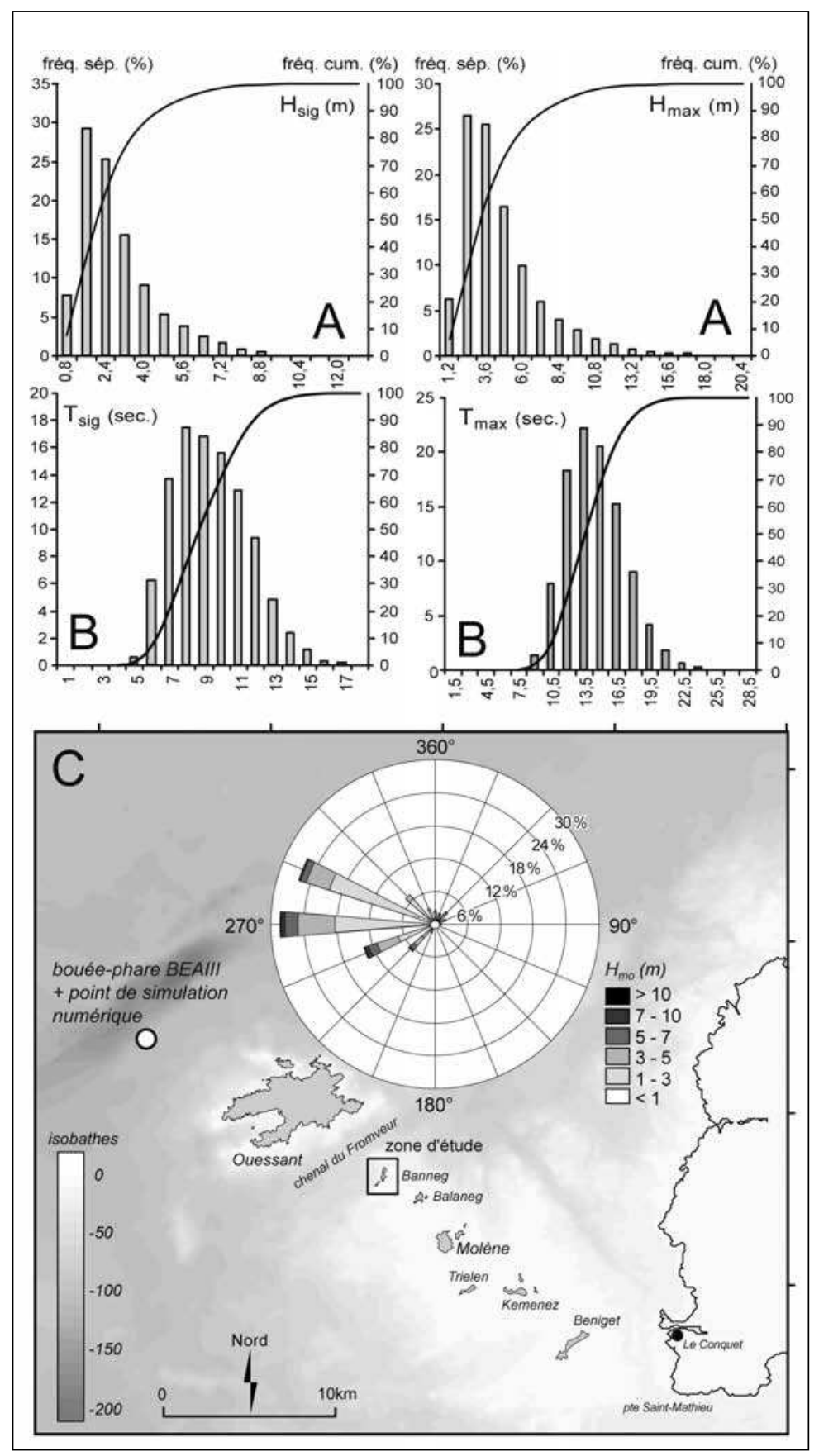

Fig. 3 - Caractéristiques hydrodynamiques de la mer d'Iroise. Histogrammes de houle. A : hauteurs significatives Hsig et hauteur maximale Hmax ; B : périodes significatives Tsig et période maximale Tmax à partir des mesures effectuées au large de Ouessant par la bouée-phare BEAlll du 30/08/1985 au 17/08/2001 (source : site Candhis du CETMEF - http://www.cetmef.equipement.gouv.fr/donnees/candhis/home.php) ; C : spectre directionnel des houles au large de Ouessant (source : simulations numériques effectuées par le LNHE-EDF avec le soutien du CETMEF).

Fig. 3-Hydrodynamic characteristics of the Iroise Sea. Histograms of significant wave heights (Hsig) and maximum wave heights (Hmax) in (A), and significant wave periods Tsig and maximum wave periods Tmax $(B)$ based on data obtained by measurements (buoy BEAIII) off Ouessant Island between 30/08/1985 and 17/08/2001 (source: site Candhis du CETMEF - http://www.cetmef.equipement.gouv.fr/donnees/candhis/home.php); C: deep-sea wave directions off Ouessant Island (source: numerical data obtained from LNHE-EDF laboratory with the help of CETMEF).

cercle concave comme au centre sud et au sud (fig. 5A). Ils forment donc des remparts ou barrages nettement étirés et plus ou moins continus. Cette disposition qui est la plus remarquable d'un point de vue morphologique est appelée dans la littérature anglo-saxone " imbricated boulder trains, continuous boulder ridges 》 ou "detrital boulder ridges » (Nott, 1997, 2000 ; Kelletat et Schellmann, 2002). Ce type d'organisation d'ensemble souffre deux exceptions. D'une part, dans le groupe du centre nord localisé dans la partie la plus basse de l'île, les empilements de blocs sont éparpillés en tous sens. D'autre part, les amas 2, 11 et 29 sont situés immédiatement au bord de la falaise, au débouché de couloirs creusés par l'érosion marine à l'emplacement de failles orientées nord-ouest. Ces couloirs ont une pente inférieure à $33 \%$, relativement faible dans ce contexte. Ils servent de rampes ascensionnelles aux blocs, en canalisant les déferlements dans des sortes de conduites forcées. Au centre et au centre sud de l'île se trouvent les accumulations les plus massives (fig. 5B) : les amas 28 et 31 forment un ensemble de plusieurs centaines de mètres cubes (fig. 4C), long de $60 \mathrm{~m}$ et large de a été construite afin d'obtenir une lecture synthétique de la topographie.

Les amas de blocs cyclopéens se trouvent sur la côte occidentale en arrière de la falaise exposée aux houles atlantiques. Ils sont situés à des altitudes comprises entre 7 et $14,5 \mathrm{~m}$ IGN 69, c'est-à-dire entre 3 et 10,5 mètres au-dessus du niveau des plus hautes mers. Comme le montre la figure $4 \mathrm{~A}$, les 52 amas recensés représentent un volume total d'environ $1000 \mathrm{~m}^{3}$, vides interstitiels compris (fig. 4C). À cela s'ajoutent des blocs isolés qui n'ont pas été cartographiés.

L'ensemble des amas a été réparti en six groupes principalement localisés au droit des rentrants de la côte, séparés par des saillants plus ou moins marqués (fig. 4A). Dans la plupart des cas, ils sont alignés soit parallèlement au trait de côte, comme au nord et au centre de l'île, soit en arc de $20 \mathrm{~m}$. C'est aussi dans ce secteur que leur disposition est la plus remarquable car ils forment localement des barrages triples parallèles mais nettement séparés les uns des autres par des étendues de roche nue ou de pelouse.

L'analyse des dépôts sur les six secteurs du nord au sud de l'île montre que le volume des blocs n'est corrélé ni avec la hauteur à laquelle ils se trouvent, ni avec la pente de la falaise (fig. 6A et 6B), ces deux paramètres ne semblant pas jouer sur l'importance des processus d'accumulation. Toutefois, si l'on considère séparément le centre de l'île, une certaine relation existe entre la pente, l'altitude et le volume des dépôts accumulés. Il n'existe pas non plus de corrélation entre la distance maximale de projection des blocs et les deux variables que sont l'altitude et la pente de la falaise (fig. 6C et 6D). Il semblerait donc que du nord au sud de 


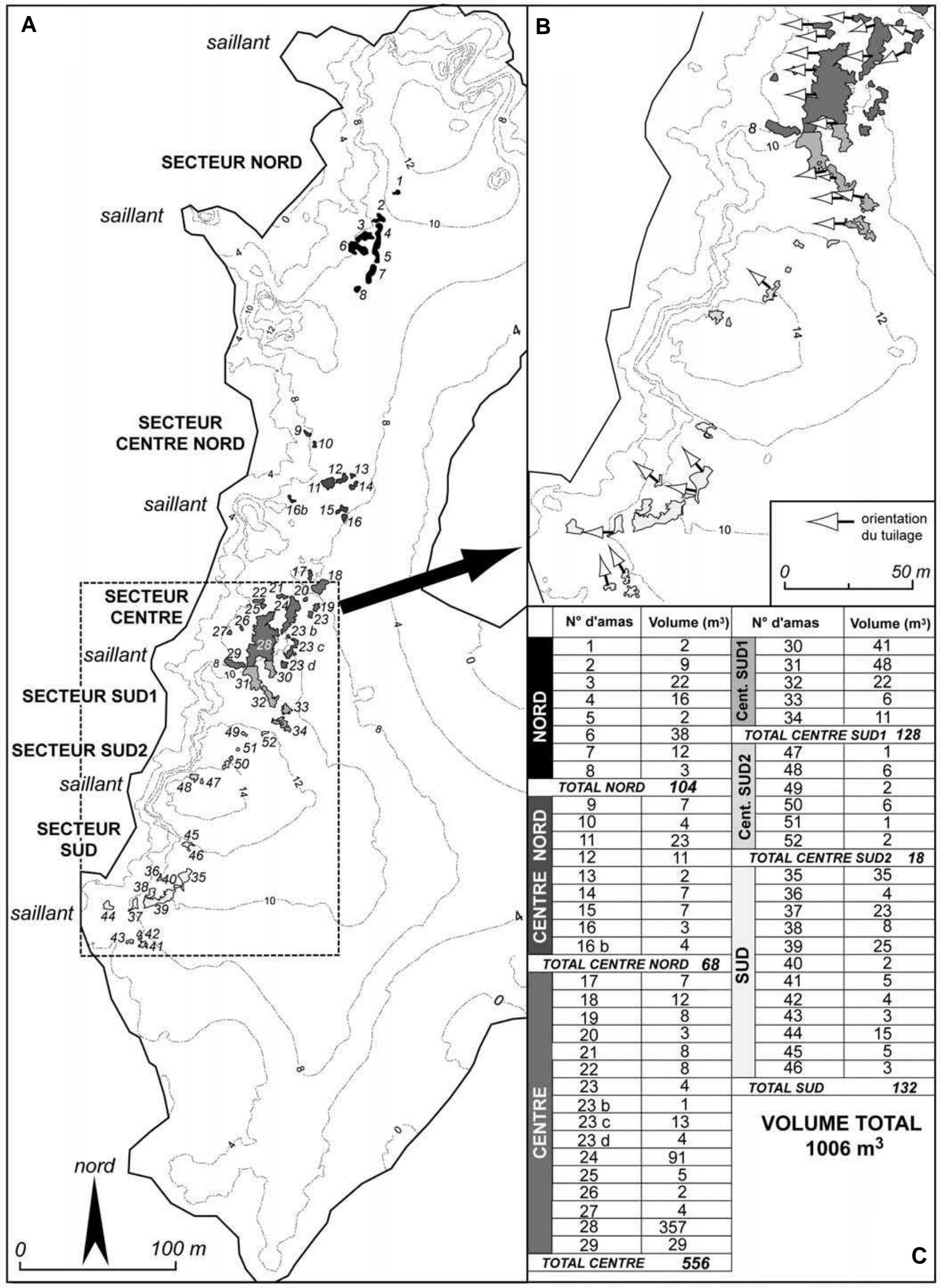


Fig. 4 - Caractéristiques des amas cyclopéens de l'île de Banneg. A : localisation ; B : direction de provenance des houles en fonction du tuilage. $C$ : volume des amas.

Fig. 4 - Characteristics of cyclopean clast deposits on Banneg Island. A: location; B: wave direction based on dip measurements; C: volume of clast deposits.

l'île, des cellules individualisées se distinguent les unes des autres par leur fonctionnement morphodynamique. Les processus hydrodynamiques sont commandés par la morphologie de la zone intertidale et des petits fonds situés en avant de la falaise ; ils conditionnent la mobilisation et les caractéristiques des dépôts de blocs telles que leur volume ou leur position. Selon les secteurs, les blocs sont soit empilés de façon anarchique, soit organisés sous forme de tuilage (fig. 5C). Dans ce cas, les dalles projetées viennent progressivement se plaquer les unes contre les autres. Comme l'ont montré plusieurs auteurs, la perpendiculaire au tuilage est un excellent indicateur de la direction de la houle incidente à l'origine de leur mise en place (Jones et Hunter, 1992 ; Noormets et al., 2002 ; Nott et Bryant, 2003 ; Nott, 2000). Les mesures effectuées indiquent une prédominance des houles de secteur ouest $\left(270^{\circ}\right)$ pour les amas du centre et centre sud1, et de secteur ouest-nord-ouest $\left(300^{\circ}\right)$ pour ceux du centre sud2 (fig. 4B). Pour les amas du sud de l'île, la direction du tuilage, bien plus complexe, suggère une prédominance des houles de secteur ouest-nord-ouest $\left(300^{\circ}\right)$ au secteur nord-ouest $\left(330^{\circ}\right)$. Dans ce secteur, la présence d'écueils et d'îlots en avant de la falaise engendre une déformation importante de la propagation des vagues à la côte.

Les blocs cyclopéens sont en majorité anguleux, contrairement à ceux de la zone intertidale qui sont constitués de très grosses boules émoussées. Il s'agit de dalles individualisées par le réseau de diaclases, qui ont été arrachées à la falaise et projetées par les vagues vers l'intérieur de l'île. Ce type de matériel appelé dans la littérature anglosaxone «large clast» (Dawson et Shi, 2000 ; Whelan et Keating, 2004), a été particulièrement bien décrit dans le cadre des recherches effectuées sur le littoral d'Hawaii (Noormets et al., 2002, 2004). Parmi les amas les plus importants, 908 blocs ont été mesurés au

Fig. 5 - Disposition morphologique des amas cyclopéens. A : disposition en arc de cercle des amas et barrages du sud de l'île de Banneg (cliché B. Fichaut, juin 2006) ; B : amas 28 situé au centre de l'île de Banneg constituant le barrage de blocs cyclopéens le plus massif (cliché $B$. Fichaut, juin 2006) ; C : disposition en tuilage des blocs de l'amas 22 indiquant la direction de la houle à l'origine de leur mise en place (cliché S. Suanez, juin 2006).

Fig. 5 - Morphological arrangement of large clasts. A: train of imbricated clasts forming an arc in the south of Banneg Island (photo B. Fichaut, June 2006); B: imbricated clast unit No. 28 located in the centre of the island and corresponding to its largest deposit (photo B. Fichaut, June 2006); C: seaward dip of imbricated clast unit No. 22 indicating wave direction linked to sedimentation processes (photo: S. Suanez, June 2006). mètre ruban afin de déterminer leur taille, leur volume et leur poids. La taille médiane avoisine $0,8 \times 0,6 \times 0,4 \mathrm{~m}$ pour un poids équivalant à 0,6 tonne, mais elle varie fortement d'un secteur à l'autre de l'île. Le plus gros bloc, 5,30 x $3,90 \times 0,50 \mathrm{~m}$, se situe au centre de l'île (amas 28) ; pesant 32 tonnes, il a été déposé à une altitude de $9 \mathrm{~m}$ et à 14 mètres en retrait de la falaise. Il existe une étroite relation entre la taille des blocs et leur position par rapport au bord

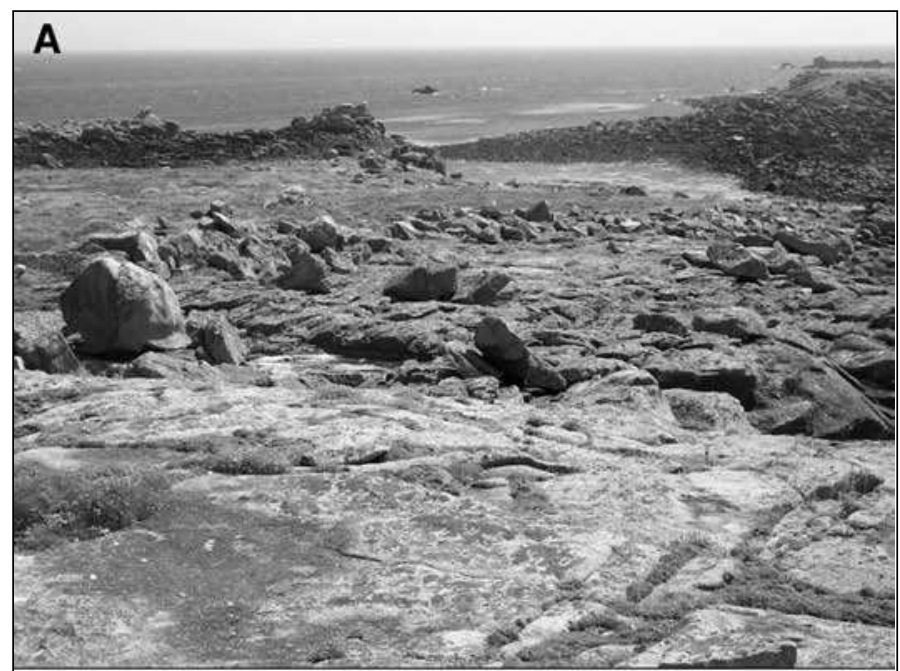

B

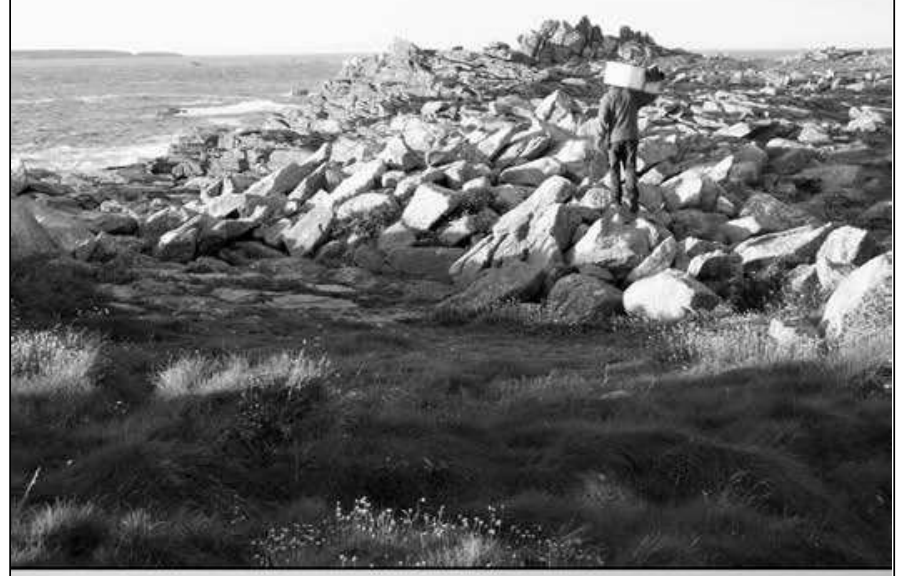

C

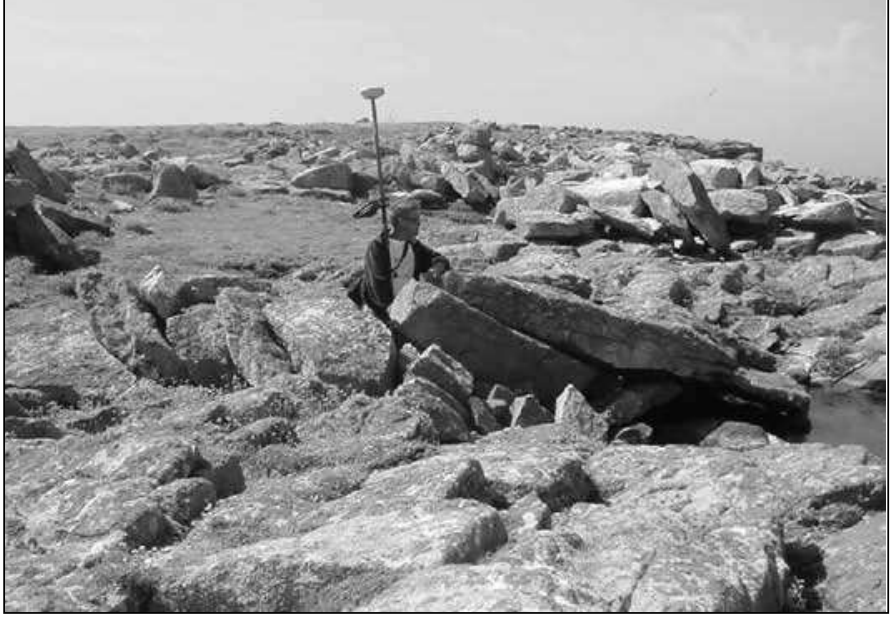



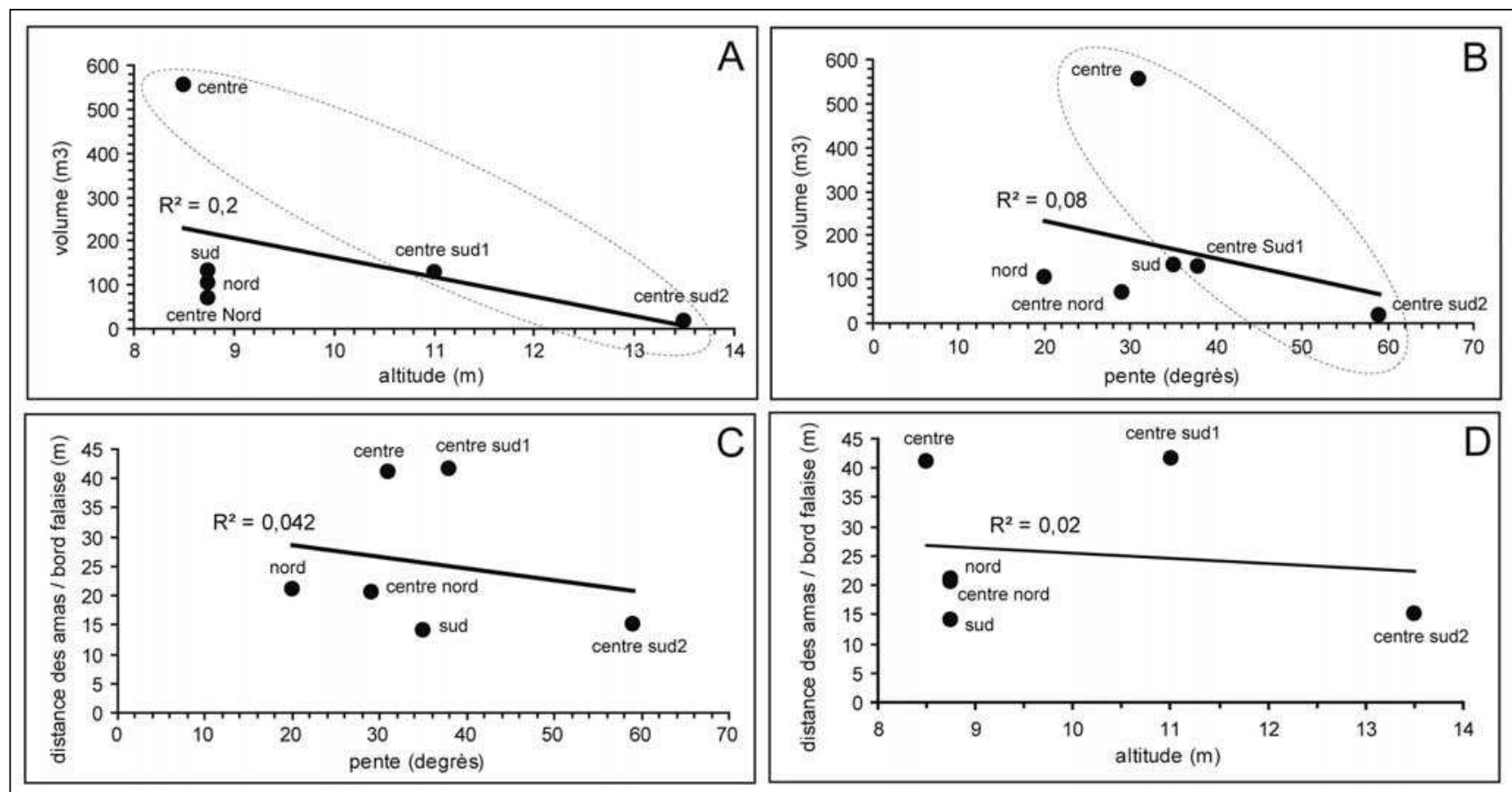

Fig. 6 - Corrélations entre les paramètres morphologiques de la falaise et les caractéristiques des amas. A : relation entre l'altitude de la falaise et le volume des amas ; B : relation entre la pente de la falaise et le volume des amas ; C : relation entre la pente de la falaise et la distance maximale de projection des blocs par rapport au bord de falaise ; $\mathrm{D}$ : relation entre altitude de la falaise et la distance maximale de projection des blocs par rapport au bord de falaise.

Fig. 6 - Correlations between sea-cliff morphological parameters and imbricated clast components. A: correlation between sea-cliff height and volume of imbricated clasts; B: correlation between slope of steep cliff and volume of imbricated clasts; C: correlation between slope of steep cliff and maximum inland distance of clasts; $D$ : correlation between sea-cliff height and maximum inland distance of clasts.

de la falaise. Plus la distance augmente, plus la taille diminue (fig. 7A). Cette relation qui s'observe en tout point est plus affirmée dans le secteur central ( $\left.\mathrm{R}^{2} 96 \%\right)$. À cet endroit les derniers des barrages triples sont constitués de blocs systématiquement plus petits que les premiers qui font face à la mer. Cette corrélation rejoint les observations faites par de nombreux auteurs (Nanayama et al., 2000 ; Dawson, 1994 ; Bryant et al., 1992), et atteste vraisemblablement d'une diminution d'énergie vers l'intérieur de l'île. L'analyse a également porté sur la relation entre la distance au bord de la falaise et le tri granulométrique des blocs, exprimée par le coefficient de variation $(\mathrm{CV})$ :

$$
C V=\frac{\sigma}{\bar{X}} \times 100
$$

où $\sigma$ correspond à l'écart type et $\bar{X}$ à la moyenne. Globalement, l'analyse statistique montre qu'aucune relation n'existe entre le tri granulométrique et la distance au bord de la falaise, à l'exception du grain médian des blocs des amas du sud de l'île (fig. 7 B et C). Dans ce cas, il semblerait que plus la distance augmente plus les blocs sont mal triés. Ces résultats vont pourtant à l'encontre des observations faites par différents auteurs montrant que les blocs sont mieux triés lorsque la distance à la côte augmente (Bourrouilh-Le Jan et Talandier, 1985 ; Nott, 1997, 2000, 2003 ; Nanayama et al., 2000).

\section{Dynamique de mise en place des amas de blocs cyclopéens}

La mise en place d'amas de blocs cyclopéens est interprétée comme le résultat du déferlement de vagues de tsunami ou de tempête (ouragans, cyclones, etc.). Dans le cas de l'archipel de Molène, comme pour la majeure partie du littoral occidental européen situé sur une marge passive où l'activité tectonique de faible énergie ne produit pas de vagues géantes, il est difficile d'attribuer ces dépôts à des tsunamis. À l'inverse, les observations menées au cours des dernières décennies ont permis de voir combien les tempêtes océaniques pouvaient engendrer des modifications morphosédimentaires aussi significatives que certains tsunamis.

Au printemps 1990, un travail d'investigation fut entrepris sur l'île de Banneg afin d'évaluer l'impact des tempêtes majeures de l'hiver 1989-1990 sur le milieu naturel (Fichaut et Hallégouët, 1989). Les observations montrèrent qu'une grande partie des accumulations en place avait été remodelée. Plusieurs centaines de tonnes de dalles avaient été arrachées aux falaises ou aux amas préexistants et projetées à l'intérieur de l'île. Les processus d'érosion et de transport les plus significatifs se produisirent dans la partie centrale. Au niveau des deux ensellements qui se tiennent entre 8 m IGN 69 (au centre nord) et 8,75 m IGN 69 (au centre), l'île fut submergée par des niveaux d'eau extrêmes supérieurs à ces alti- 


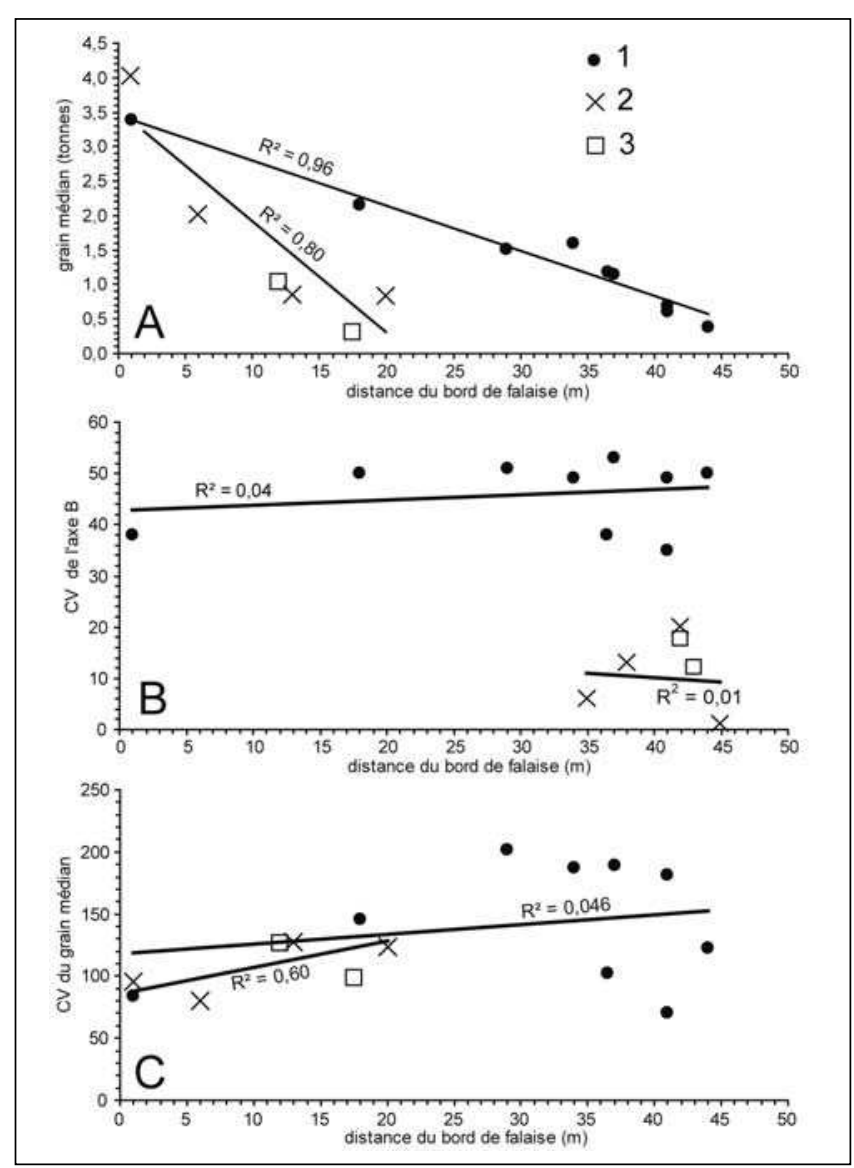

Fig. 7 - Corrélation entre les paramètres granulométriques et les caractéristiques des amas. $A$ : relation entre la taille des blocs et la distance par rapport au bord de la falaise ; $B$ et $C$ : relation entre le tri des blocs et la distance par rapport au bord de la falaise ; 1 : amas du centre de l'île ; 2 : amas du sud de lî̀le ; 3 : amas du nord de l'île.

Fig. 7 - Correlation between sedimentary parameters and imbricated clast components. A: correlation between the size of clasts and the distance from cliff edge $B$ and $C$ : correlation between sorting and the distance from cliff edge; 1 : imbricated clast in the central part of the island; 2: imbricated clast in the south part of the island; 3 : imbricated clast in the north part of the island. tudes. L'eau issue des déferlements s'écoula d'ouest en est, en creusant des formes en vallon jusqu'à la roche en place. Là, les blocs entraînés par l'écoulement torrentiel de la lame d'eau furent déposés sous forme d'amas isolés, voire transportés jusqu'à la côte est de l'île (fig. 8). Ailleurs, les écoulements ne firent que décaper le sol sur des surfaces qui ont depuis été recolonisées par une pelouse aérohaline. Plus récemment, en décembre 2005, nous avons constaté que quelques blocs avaient été arrachés aux falaises. L'un d'eux, d'un poids de 2,6 tonnes $(1,7 \times 1 \times 0,5 \mathrm{~m})$, enlevé à $10 \mathrm{~m}$ d'altitude a rebondi sur une tête de roche à 11,6 m d'altitude, avant de se déposer près de l'amas 22 après un trajet de $12 \mathrm{~m}$. Ces déplacements ont été attribués aux tempêtes survenues entre le 30 octobre et le 7 novembre 2005.

\section{Caractéristiques hydrodynamiques à l'origine de leurs déplacements}

L'étude des conditions hydrodynamiques permettant d'expliquer ces déplacements a été faite à partir des mesures réalisées durant les tempêtes de l'hiver 1989-1990. Elle s'est appuyée sur trois types de données : 1) des données météo-marines acquises auprès de Météo France sur le vent (direction et vitesse) et la pression barométrique enregistrés au sémaphore du phare de Créac'h sur l'île de Ouessant ; 2) les états de la mer, à savoir les données de houle obtenues d'une part, à partir des mesures in situ par la bouée-phare BEAIII et fournies par le CETMEF (Plouzané) et, d'auter part, par simulations numériques calculées au même point et pour la même période, par EDF-LNHE (Chatou) ; 3) la marée enregistrée au Conquet et fournie par le SHOM (Brest). Dans un premier temps, l'analyse a porté sur le choix des données de houles à retenir en sachant qu'il existe un biais négatif pour la mesure in situ (Benoit et al., 2006). Comme nous l'avons souligné plus haut, ces auteurs ont montré que les données enregistrées par la bouée-phare BEAIII sous-estiment les plus fortes hauteurs significatives pour les événements mor-

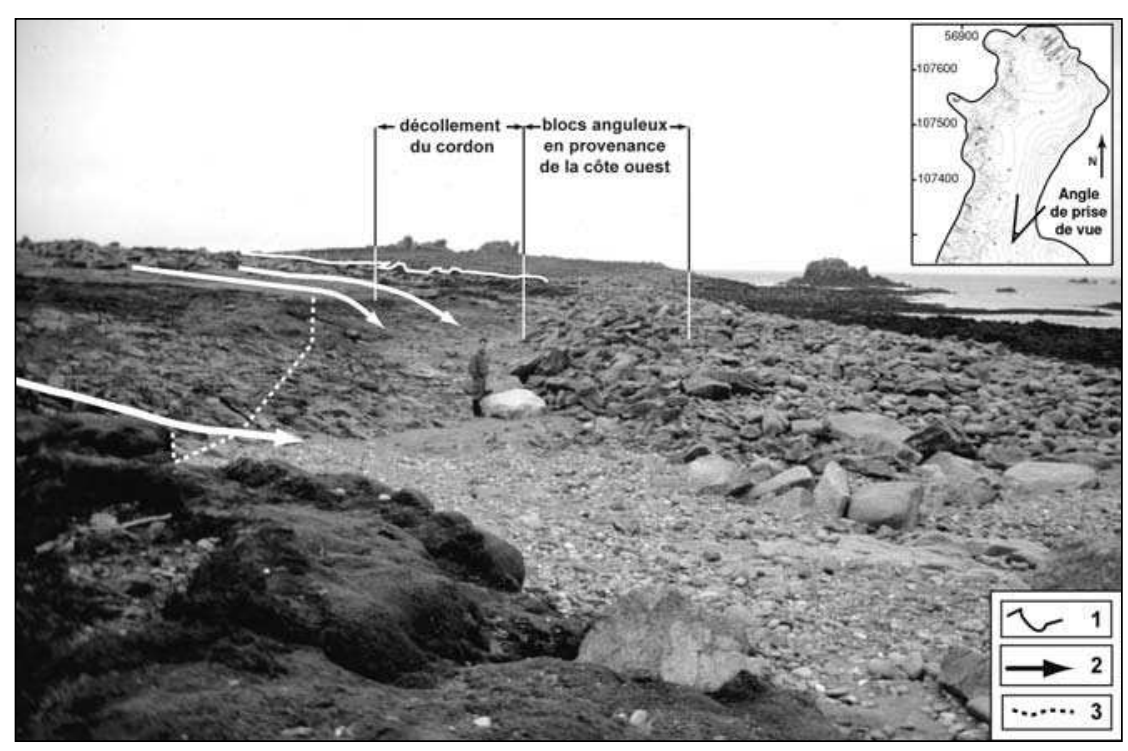

Fig. 8 - Littoral oriental de l'île de Banneg au droit des secteurs centre et centre nord. La limite de végétation arrachée (1) montre clairement la puissance des écoulements torrentiels qui ont traversé l'île d'ouest en est (2) et qui ont aussi creusé la gorge jusqu'à la roche en place (3). Le cordon de galets a été décollé du trait de côte par l'écoulement ayant charrié des blocs anguleux venant de l'ouest, qui ont été incorporés dans l'édifice (cliché B. Hallégouët, avril 1990).

Fig. 8 - Eastern shoreline in the central and north-central areas of Banneg island. The limit of degraded vegetation (1) clearly shows the power of torrential flows, which crossed the island from west to east (2) and cut a gorge down to the bedrock (3). The shingle beach ridge was shifted seaward from the shoreline by flows that also transported angular blocks from the western side. The blocks were incorporated into the shingle beach ridge (photo $B$. Hallégouët, April 1990). 


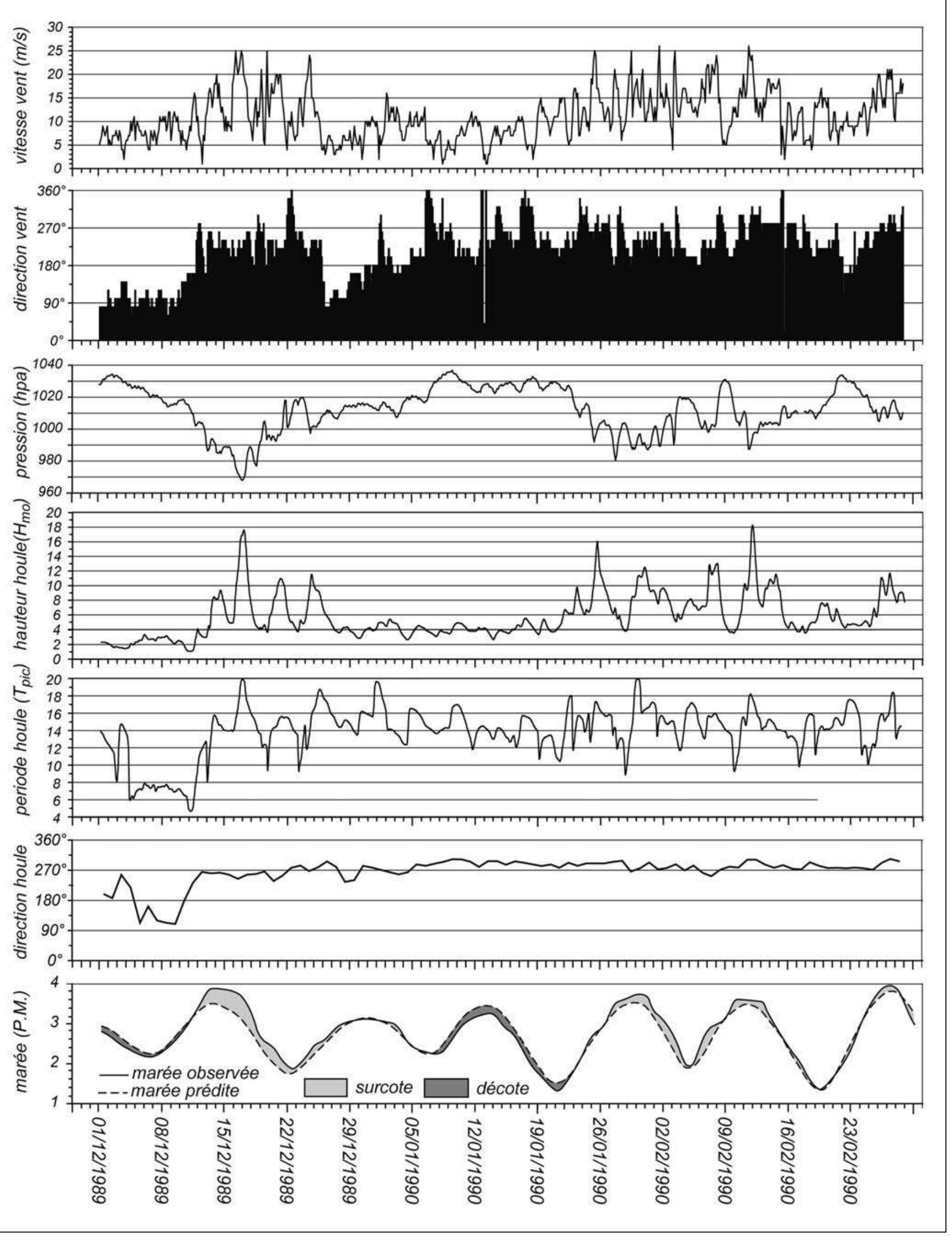

Fig. 9 - Caractéristiques météorologiques et océaniques (houle, vent, pression barométrique et surcote) des tempêtes de l'hiver 1989-1990.

Fig. 9 - Meteorological and oceanic characteristics (wave, wind, pressure and surge) recorded during the 1989-1990 winter storms. 
phogènes extrêmes du fait du poids considérable de la bouée (80 tonnes). C'est la raison pour laquelle nous avons retenu les données acquises par simulation numérique. La compilation de l'ensemble des données météo-marines révèle que trois épisodes tempétueux apparaissent clairement entre le $1^{\text {er }}$ décembre 1989 et le 28 février 1990 (fig. 9). Le premier, du 13 au 27 décembre 1989, est marqué par des hauteurs de houles atteignant $18 \mathrm{~m}$ et des surcotes proches de $90 \mathrm{~cm}$. Durant cet épisode, les pressions tombées à $970 \mathrm{hPa}$ ont engendré des vents de $25 \mathrm{~m} / \mathrm{s}$. Lors du second événement, du 23 janvier au 14 février 1990, on enregistre à deux reprises des hauteurs de houle comprises entre 16 et $18 \mathrm{~m}$ combinées à des vents dépassant cinq fois $25 \mathrm{~m} / \mathrm{s}$. Les pressions un peu moins creusées $(985 \mathrm{hPa})$ sont associées à des surcotes moins importantes de l'ordre de $50 \mathrm{~cm}$. Le dernier épisode survenu à la fin février 1990 a engendré des hauteurs de vagues plus faibles $\left(H_{m o} 12 \mathrm{~m}\right)$; en revanche, les niveaux de pleine mer sont les plus importants de toute la période étudiée, avec une marée observée atteignant 4 m IGN 69.

En second lieu, nous avons estimé les niveaux d'eau extrêmes afin de savoir si la hauteur maximale de la tranche d'eau au déferlement avait pu atteindre des altitudes similaires, entre 7 et 14,5 m IGN 69, à celles des amas de blocs présents sur le terrain. Pour ce faire, deux paramètres agissant sur la déformation du plan d'eau à la côte ont été considérés. Le premier qualifié de " marée de tempête » (Caspar et al., 2007) résulte de l'addition d'une surcote d'origine atmosphérique à la marée astronomique : cette valeur est donnée par la marée observée. À ce paramètre se surimpose l'action de la houle à la côte dont un des processus appelé run-up est défini comme la somme de deux composantes : les variations du plan d'eau liées à l'agitation (setup : $\bar{\eta}_{\max }$ ) et la hauteur du jet de rive (wave uprush : $R$ ), soit $R^{T}=\bar{\eta}_{\text {max }}+R$. Le run-up a été calculé à partir des équations de H. Mase (1989) applicables pour des pentes d'avant plage ( $\tan \beta$ comprises entre 0,03 et 0,2 (de 3 à $20 \%)$, et des cambrures de houles en eau profondes $\left(H_{o} / L_{o}\right)$ allant de 0,007 à 0,07 :

$$
\begin{aligned}
& \frac{R^{T}{ }_{\text {max }}}{H_{\text {sig }}}=2,32 \xi_{o}^{0,77} \\
& \frac{R^{T}{ }_{2 \%}}{H_{\text {sig }}}=1,86 \xi_{o}^{0,71}
\end{aligned}
$$

où $R^{T}{ }_{\text {max }}$ correspond au run-up maximum et $R^{T} \%$ à celui dépassé par $2 \%$ des plus hauts jets de rive ; $H_{\text {sig }}$ : hauteur significative au large (en mètres) ; constante $C=2,32$ pour $R^{T}{ }_{\text {max }}$ et 1,86 pour $R^{T}{ }_{2 \%} ; \xi_{o}$ : nombre d'Iribarren (Battjes, 1974) :

$$
\xi_{o}=\frac{\tan \beta}{\left(H_{s i g} / L_{o}\right)^{1 / 2}}
$$

Avec des pentes de l'avant côte $(\tan \beta)$ - pour des profondeurs comprises entre -5 et $-40 \mathrm{~m} \mathrm{NGF}$ - allant de $0,02 \mathrm{au}$ nord et au centre nord, et 0,05 au centre et au sud de l'île, et des valeurs de cambrure moyennes annuelles évoluant entre 0,014 et 0,016 durant la période 1979-2002, les caractéristiques de notre site d'étude remplissaient les conditions d'utilisation de ces équations. Pour des pentes variables du nord au sud de l'île, le run-up $R^{T}{ }_{2 \%}$ atteint respectivement $7 \mathrm{~m}$ et 13,5 m. Les valeurs maximum $R^{T}$ max sont respectivement égales à $7,7 \mathrm{~m}$ et $15,7 \mathrm{~m}$.

L'addition des hauteurs de la marée observée et du run-up a permis d'estimer les niveaux extrêmes enregistrés durant les trois mois (fig. 10). Les hauteurs d'eau maximum $\left(R_{\text {max }}^{T}\right)$ ont atteint respectivement $11,15 \mathrm{~m}$ au nord et centre nord et 18,91 $\mathrm{m}$ au centre et au sud de l'île le 16 décembre 1989. Ces valeurs, qui ne représentent que des ordres de grandeur, s'avèrent en partie valables lorsqu'on les confronte aux observations de terrain effectuées par B. Fichaut et B. Hallégouët (1989). La figure 10A montre que les niveaux d'eau ont à peine dépassé la hauteur des falaises au nord et au centre nord de l'île à trois reprises, ce qui suppose des débordements peu étendus. Ceci est en accord avec les observations de terrain effectuées au nord de l'île indiquant que la zone d'arrachement de la pelouse aérohaline avait été limitée à une dizaine de mètres en arrière du bord de la falaise (fig. 11). À l'inverse, l'absence de débordements importants au centre nord va à l'encontre de ces résultats lorsque l'on sait que des écoulements tumultueux induits par une submersion marine importante, ont affecté ce secteur d'ouest en est (fig. 8). Au centre et au sud de l'île, des niveaux d'eau largement supérieurs à l'altitude des falaises ont engendré des débordements plus fréquents (fig. 10B), ce qui pourrait expliquer l'importance des accumulations dans ces secteurs. Au centre, ces résultats ne semblent pas aberrants car, comme nous l'avons vu, les impacts de la submersion marine ont considérablement modifié le milieu biologique et morphologique (fig. 8). En revanche, et à nouveau, ce schéma ne s'applique pas aussi clairement dans le sud de l'île, car le volume des accumulations reste modeste alors que l'altitude des falaises est bien inférieure à la hauteur des niveaux d'eau. Il est ainsi difficile d'expliquer la particularité des secteurs tels que le centre nord ou le sud de l'île. Enfin, la figure 10B montre que les hauteurs d'eau les plus importantes atteintes au centre de l'île $(18,91 \mathrm{~m})$ restent bien supérieures à l'altitude maximale des blocs que l'on trouve dans ce secteur (14,5 $\mathrm{m}$ au centre sud2). Ce fait va dans le même sens que les observations effectuées par de nombreux auteurs qui ont montré que, bien souvent, la limite supérieure des dépôts se situe sous le niveau maximum atteint par le run-up (Dawson, 1994).

\section{Faible fréquence des épisodes morphogènes depuis 1979}

Un inventaire des épisodes morphogènes, susceptibles d'arracher ou de déplacer des blocs, a été dressé pour la période couvrant les vingt-quatre dernières années (19792002). La méthode d'analyse mise en œuvre est identique à celle qui a été retenue pour les tempêtes de l'hiver 19891990, la marée observée ayant été additionnée au run-up. Ce dernier paramètre a été calculé à partir des données de houle obtenues par simulation numérique par EDF-LNHE du 1er janvier 1979 au 31 août 2002, période également retenue pour la marée enregistrée au Conquet. Toutefois, cette série 


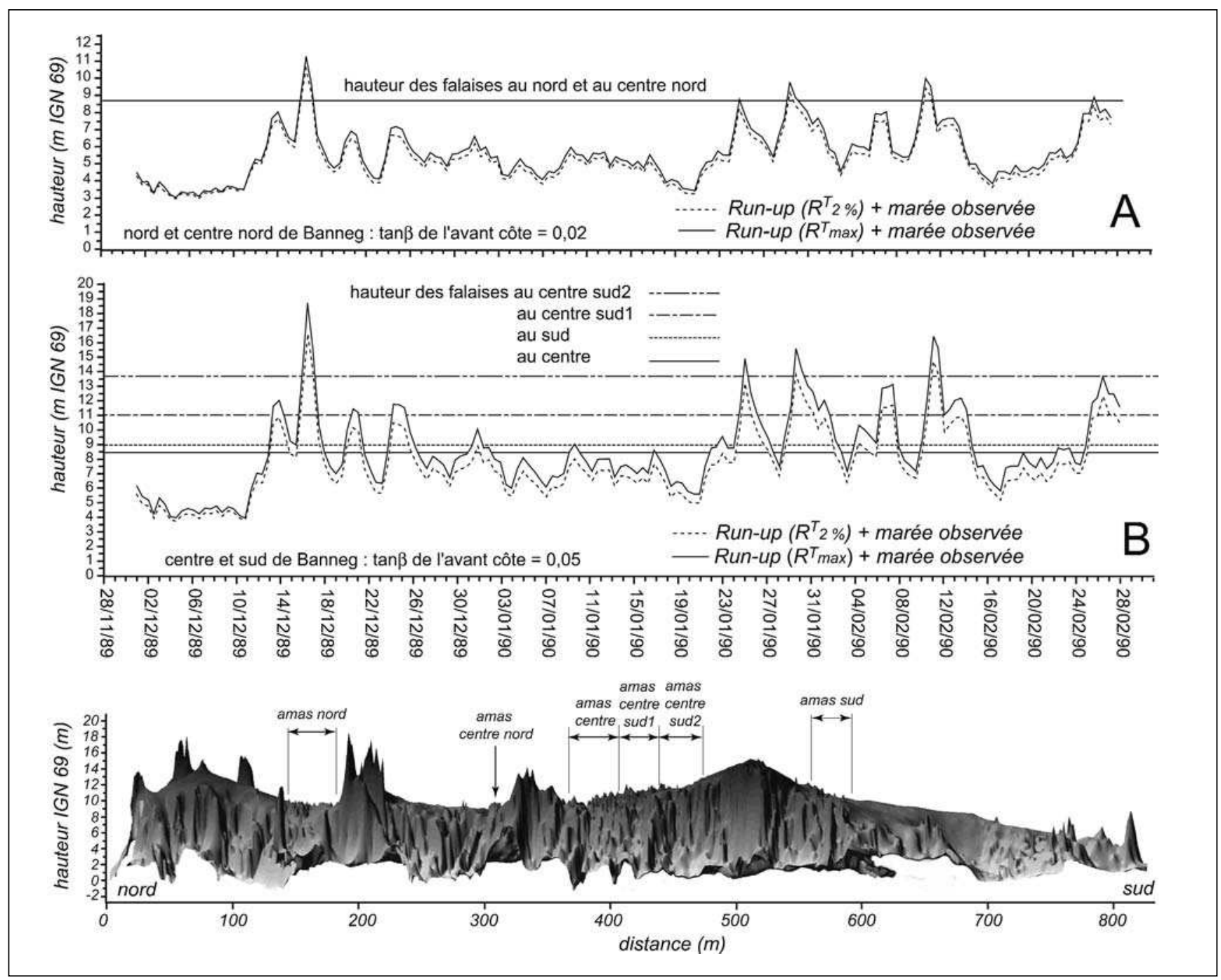

Fig. 10 - Hauteurs calculées des niveaux d'eau extrêmes (marée observée et run-up) par rapport à l'altitude des falaises durant les tempêtes de l'hiver 1989-1990.

Fig. 10 - Heights of extreme water levels (observed tide and run-up) compared to sea-cliff heights during the 1989-1990 winter storms.

comportait des lacunes plus ou moins importantes ; elles ont été comblées par les données marégraphiques de Brest même si, par rapport au Conquet, la différence de hauteur d'eau pour une marée prédite de vive-eau est de $20 \mathrm{~cm}$ et que le décalage horaire des étales de marée est de 5 minutes. Ceci étant, les corrélations établies afin de tester la réponse similaire ou non des deux sites aux forçages météorologiques (phénomènes de surcotes et de décotes) ont indiqué une bonne relation entre Brest et Le Conquet : $\mathrm{R}^{2}$ est compris entre 80 et $90 \%$ suivant les périodes retenues par l'analyse statistique. Les biais les plus importants relevés d'un site à l'autre ont été observés durant l'hiver et s'expliquent par le fait que Brest, en fond de rade, est beaucoup plus protégé des flux météo-océaniques que Le Conquet directement ouvert sur la mer d'Iroise.

La figure 12 illustre les résultats obtenus par cette analyse. La forte cyclicité caractérisant la courbe est essentiellement due aux variations saisonnières (été / hiver) des caractéristiques de la houle. Les crêtes sont conditionnées par les fortes houles de tempêtes hivernales (décembre à février), tandis que les creux s'expliquent par la diminution de la hauteur et de la période des vagues estivales. Cet inventaire montre également une variabilité des niveaux d'eau extrêmes selon des cycles supérieurs à 10-12 ans. Ainsi, la période 1979-1990 est marquée par une fréquence plus importante d'événements morphogènes que la suivante (19912002), à l'exception de l'épisode du mois de décembre 1997. Des cycles similaires, de 11 à 16 ans affectant l'évolution des surcotes à Brest entre 1860 et 1995, ont été observés par R. Bouligand et P. A. Pirazzoli (1999). Expliqués par des changements de fréquence des vents de surcote au cours des dernières décennies (Pirazzoli, 2000), ces cycles pourraient être liés à l'alternance des phases positives et négatives de l'indice d'oscillation nord atlantique ONA (Pirazzoli et al., 2004), bien que certains auteurs aient indiqué que cet indice ne pouvait à lui seul expliquer ces changements (Betts et al., 2004). Cette diminution des épisodes morphogènes pour la décennie 1990-2002 a été par ailleurs 
Fig. 11 - Perturbations biologiques et morphologiques relevées au nord de l'île après les tempêtes de l'hiver 1989-1990. 1 : limite d'arrachement de la végétation et d'érosion du sol ; 2 : isoligne $9 \mathrm{~m} ; 3$ : blocs apportés pendant les tempêtes ; 4 : cicatrices des blocs arrachés par les tempêtes (cliché B. Hallégouët, avril 1990).

Fig. 11 - Biological perturbations and morphological changes observed in the northern part of the island after the 1989-1990 winter storms. 1: limit of vegetation degradation and soil erosion; 2: $9 \mathrm{~m}$ contour interval; 3: clast deposits brought during storms; 4: scars of clasts dislodged during storms (photo B. Hallégouët, April 1990).

observée dans une étude récente portant sur la mobilité du rivage dans le sud Finistère (Hallégouët et Hénaff, 2006). Ces auteurs ont montré que depuis l'été 1990 l'ensemble des côtes d'accumulation comprises entre la pointe du Raz et l'Ile-Tudy avait connu une phase d'engraissement attribuée en majeure partie à l'absence d'événements érosifs, contrairement à la

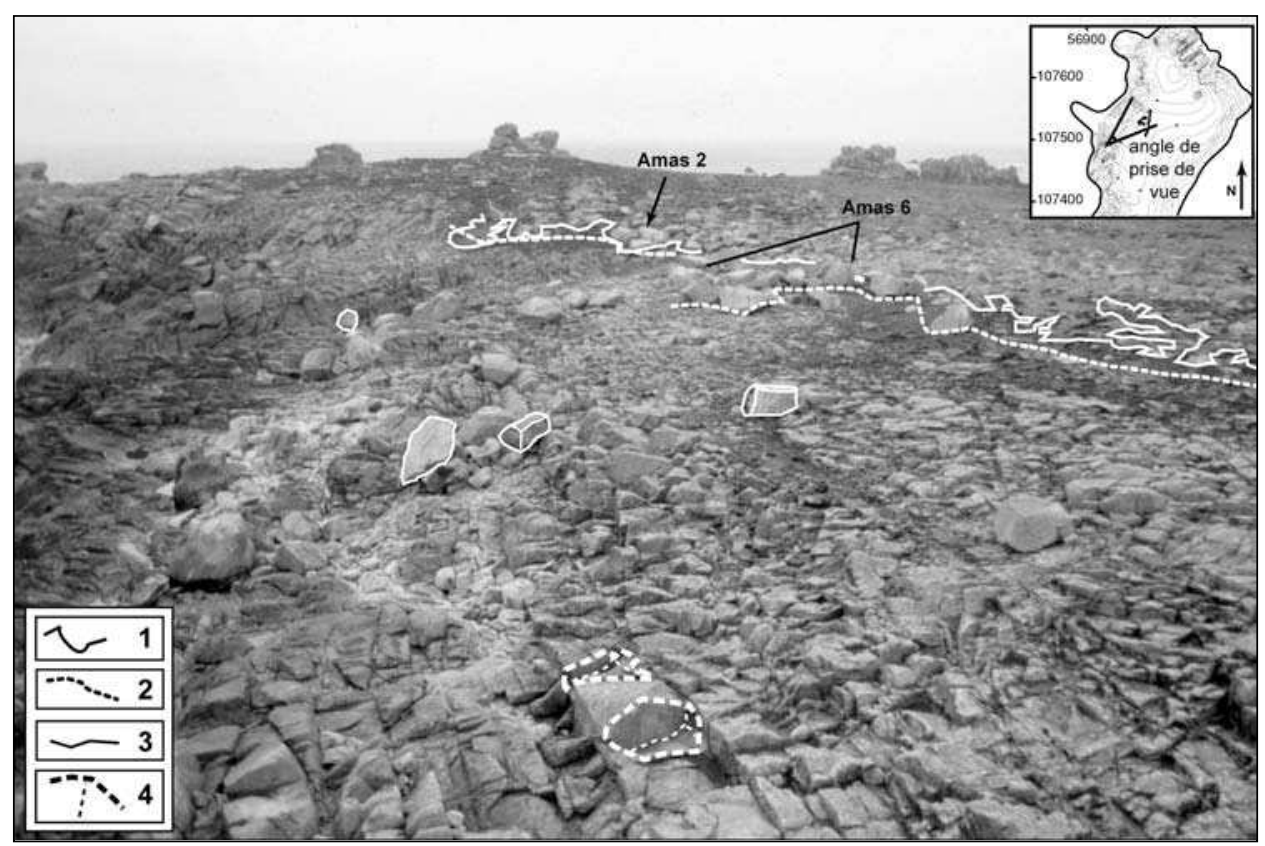

période précédente (1974 à 1989-1990), marquée pas une succession de violentes tempêtes dévastatrices.

La seconde observation montre que de 1979 à 2002, aucun épisode aussi efficace sur le plan morphogénique que les événements de l'hiver 1989-1990 n'a été recensé, même si l'hiver

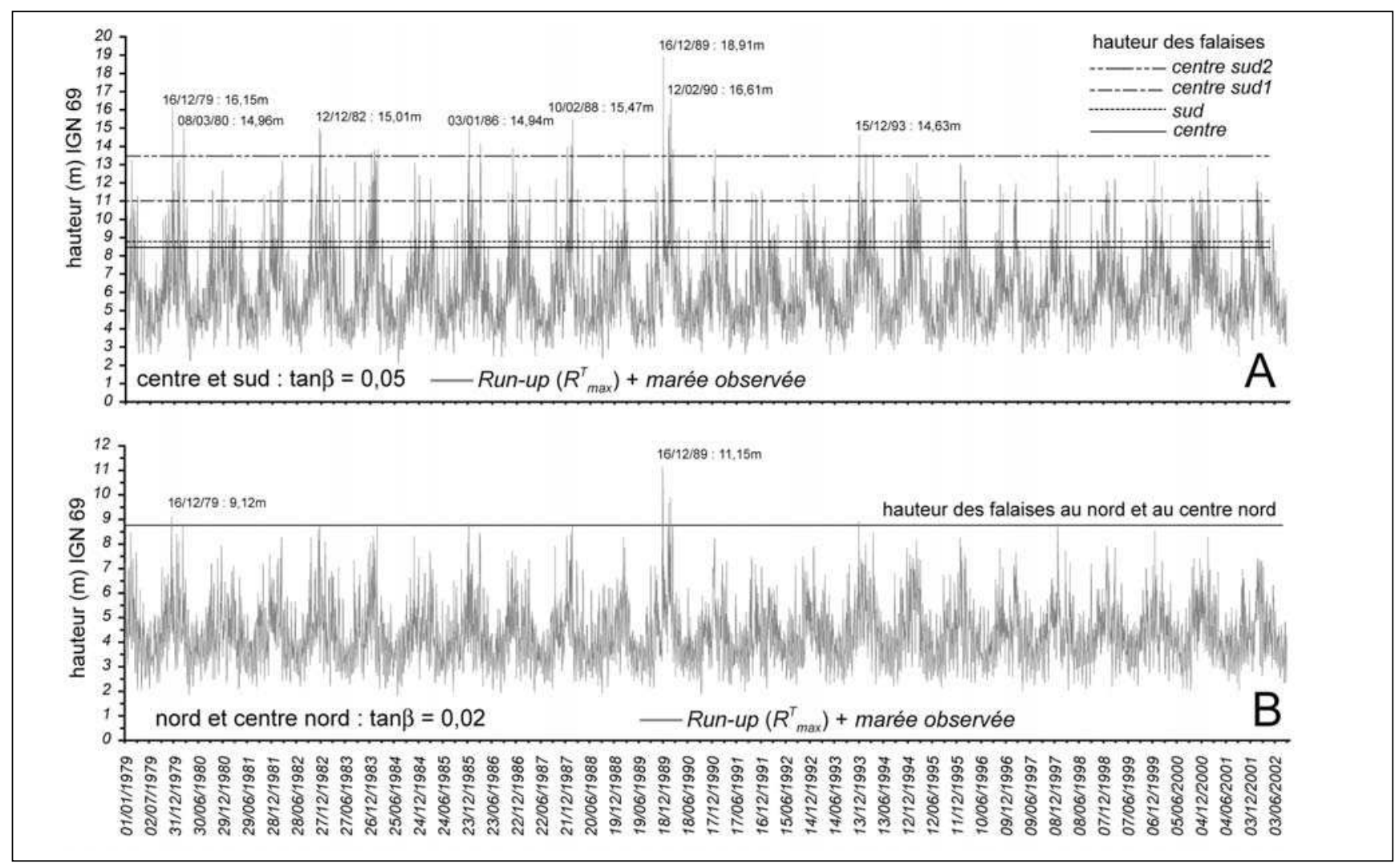

Fig. 12 - Hauteurs calculées des niveaux d'eau extrêmes (marée observée et run-up) par rapport à l'altitude des falaises pour la période du 1er janvier 1979 au 31 août 2002.

Fig. 12 - Heights of extreme water levels (observed tide and run-up) compared to sea-cliff heights for the time interval between January 1st, 1979 and August 31st, 2002. 
1979-1980 a connu plusieurs tempêtes de forte intensité. De 1979 à 1990, les épisodes de submersion des falaises sont particulièrement nombreux au centre et au sud de l'île (fig. 12A). La période suivante est marquée par une diminution de la fréquence des événements extrêmes, particulièrement après l'hiver 1997. Les processus dynamiques qui en découlent sont bien différents au nord et au centre nord de l'île où, à l'exception des tempêtes de l'hiver 1979-1980 et de 1989-1990, le franchissement des falaises par les niveaux d'eau extrêmes paraît insignifiant sur l'ensemble de la période (fig. 12B). Là encore, l'approche théorique mise en œuvre dans cette étude minimise probablement les processus dynamiques qui ont affecté le centre nord de l'île. En effet, la photo-interprétation de clichés obliques pris en 1980 et en 2005 indique que dans toute la partie centrale de l'île la morphologie des amas a été profondément modifiée (fig. 13).

\section{Discussion et conclusion}

Cette étude apporte des données nouvelles dans un domaine de recherche encore peu exploré en géomorphologie littorale française. Cela tient au fait que les blocs cyclopéens ont très longtemps été attribués aux tsunamis, sur les côtes des façades ou des îles du Pacifique soumises à de fréquents séismes. L'étude de l'île de Banneg confirme l'idée souvent débattue suivant laquelle les fortes tempêtes océaniques peuvent également engendrer des processus morphosédimentaires similaires à ceux des tsunamis (Einsele et al., 1996 ; Nott, 1997, 2003). Néanmoins, au terme de ce travail, plusieurs interrogations demeurent et ouvrent des perspectives de recherche.

Les caractéristiques morphométriques et granulométriques des blocs cyclopéens de l'île de Banneg montrent de fortes similitudes avec les résultats obtenus sur d'autres sites. La relation entre la diminution de la taille des blocs et la distance au rivage est une constante décrite par la plupart des auteurs (Nanayama et al., 2000 ; Bourrouilh-Le Jan et Talandier, 1985 ; Nott, 1997, 2000, 2003 ; Moore et Moore, 1984 ; Bryant et al., 1992 ; Scheffers et Kelletat, 2004 ; Goff et al., 2006). À l'inverse, la corrélation positive entre le tri et la distance au rivage, observée dans bien des cas, n'est pas vérifiée ici. Lorsqu'elle existe, la diminution de la taille des grains atteste d'une déperdition de l'énergie de la tranche d'eau, comme c'est le cas lors d'un seul événement extrême tel qu'un tsunami (Nanayama et al., 2000). À Banneg, ce schéma est brouillé par la superposition de

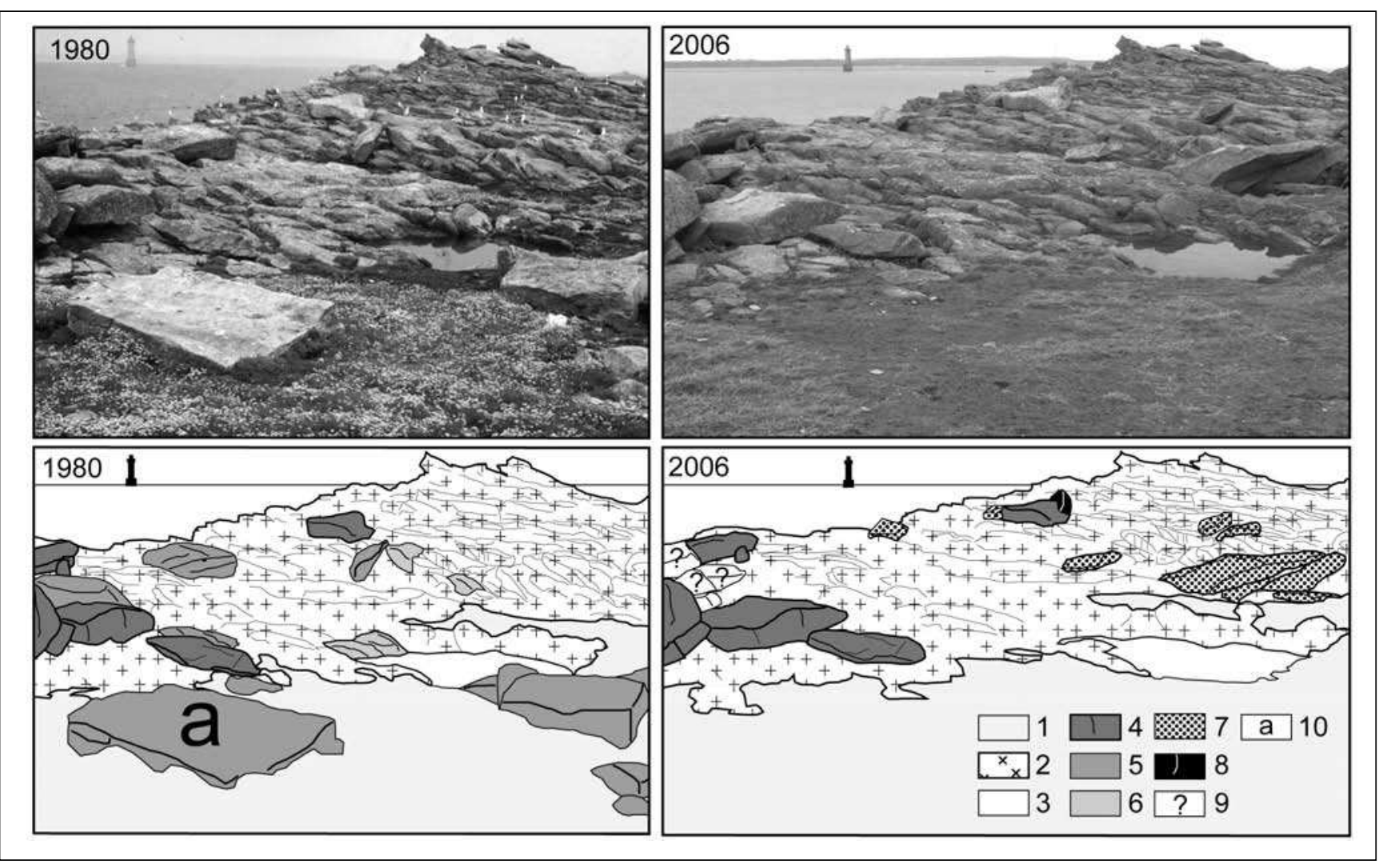

Fig. 13 - Illustration des changements morphologiques enregistrés au centre de l'île de Banneg entre 1980 et 2005.1 : pelouse aérohaline ; 2 : substrat rocheux affleurant ; $3:$ mer, mare ; 4 : blocs en place au même endroit en 1980 et $2006 ; 5:$ blocs présents en 1980 , absents en $2006 ; 6$ : dalles rocheuses arrachées entre 1980 et $2006 ; 7:$ blocs absents en $1980 ; 8:$ blocs déplacés en $2005 ; 9:$ statut indéterminé ; 10 : dalle déposée lors des tempêtes de l'hiver 1979 (d'après clichés B. Hallégouët, 1980 et B. Fichaut, 2006).

Fig. 13 - Illustration of morphological changes observed in the central part of Banneg island between 1980 and 2006.1 : grass; 2 : bedrock; 3: sea, ponds; 4: clasts located at the same place in 1980 and 2006; 5: clasts existing in 1980, absent in 2006; 6: tabular clasts detached from the bedrock between 1980 and 2006; 7: clasts absent in 1980; 8: clast moved in 2005; 9: undetermined; 10: clast deposited during the 1979 winter storms (from photos B. Hallégouët, 1980 and B. Fichaut, 2006). 
plusieurs épisodes d'intensité variable, susceptibles de remanier les amas et de mélanger des blocs de toutes tailles. Il est certain que le recours à la datation des blocs permettrait d'aller plus loin dans la compréhension de ces processus dynamiques, mais il s'avère difficile à mettre en œuvre car peu d'éléments datables sont disponibles. À première vue, la datation radiocarbone ${ }^{14} \mathrm{C}$ le plus souvent utilisée sur des coquilles prises dans des laisses de tempête (Dawson, 1994 ; Clague et al., 1999 ; Nichol et al., 2003), des concrétions coralliennes sur des blocs (Jones et Hunter, 1992 ; Kelletat et Schellmann, 2002 ; Nott et Bryant, 2003 ; Whelan et Keating, 2004) ou des niveaux vaso-tourbeux intercalés dans les dépôts grossiers (Liu et Fearn, 1993 ; Dawson, 1994 ; Bondevik et al., 2005), serait inexploitable dans le cas de Banneg car il n'existe aucun élément carbonaté directement ou indirectement rattaché aux dépôts. D'autres méthodes comme la datation par thermoluminescence, utilisée sur des sables de laisses associés à des blocs, ont donné de bons résultats (Young et al., 1996). Cette piste paraît plus appropriée si l'on tient compte du fait que des placages sableux tapissent partiellement les petits fonds situés en avant des falaises. Une troisième approche, plus efficace, serait de recourir à l'analyse lichénométrique d'espèces comme Xanthoria parietina et Ramalina siliquosa abondantes sur les blocs. Bien que cette approche n'ait été utilisée qu'en domaine continental, en morphologie fluviale (Jacob et al., 2006), en dynamique de versant (Winchester et Chaujar, 2002), ou plus couramment en domaine proglaciaire (Evans et al., 1999 ; Winchester et Harrison, 2000), certains auteurs ont montré qu'elle pouvait être aussi appliquée en milieu marin, notamment pour caractériser la mobilité de certains cordons de galets littoraux (Morel, 1997).

L'analyse menée sur les conditions hydrodynamiques de la mise en place de ces dépôts reste insuffisante sur deux points. Tout d'abord, l'usage de la formule du run-up de H. Mase (1989) ne permet pas d'expliquer les caractéristiques morphodynamiques et sédimentaires du centre nord et du sud de l'île. L'étude conduite sur l'île de Oahu (Hawaii) par R. Noormets et al. (2002) a montré le rôle important de la bathymétrie dans les différences observées entre les processus hydrodynamiques agissant d'un secteur à l'autre de l'île. Aussi, en collaboration avec le SHOM, une campagne de mesure des paramètres hydrodynamiques au déferlement (houle, marée, courants) a été envisagée pour l'hiver 2008-2009 afin de valider les résultats obtenus pour chaque secteur. Le second point faible se rapporte au fait que la période retenue pour le rétrocalcul, ne permet d'analyser que les modifications dues aux événements très morphogènes de l'hiver 1989-1990. Cette approche n'explique pas la mise en place initiale des amas, ni la présence des barrages triples que l'on trouve au centre de l'île et dont on ne sait s'ils ont été construits en un seul ou en plusieurs épisodes. Seule une étude de chroniques historiques plus longues permettrait d'établir une chronologie acceptable des événements morphogènes anciens.

Enfin, ces blocs cyclopéens correspondent à des dalles arrachées au sommet des falaises, dans la roche en place, suivant des plans de fracturation qui conditionnent probablement l'intensité et l'importance du débitage. Dans leur étude de l'archipel des Tuamotu, F. G. Bourrouilh-Le Jan et J. Talandier (1985) ont mis en lumière le rôle de la structure dans la dynamique d'arrachage des dalles calcaires, en soulignant que la localisation précise du réseau de fractures limitait l'action mécanique de la houle à un petit nombre de secteurs. Des observations similaires ont été effectuées sur l'île Grand Cayman dans les Caraïbes (Jones et Hunter, 1992) et sur l'île de Oahu à Hawaii (Noormets et al., 2002), où les zones subissant davantage l'arrachage de blocs correspondent à des secteurs fragilisés par un réseau très dense de diaclases élargies par l'érosion biochimique. On peut en déduire que les différences de calibre des blocs et de volume sédimentaire enregistrées d'un secteur à l'autre de l'île de Banneg trouvent aussi leur origine dans ce paramètre litho-structural. Pour le démontrer il s'agira d'abord d'établir une cartographie exhaustive de la fracturation en utilisant la méthode de suivi par quadra, utilisée entre autres par les botanistes, puis il faudra analyser spécifiquement les caractéristiques de résistance physique et mécanique de la roche à l'action de la houle (Noormets et al., 2004).

En définitif, les blocs cyclopéens de l'île de Banneg représentent les formes d'accumulation les plus spectaculaires de tout l'archipel de Molène, principalement constituées de formes «fuyantes » comme des queues de comètes ou des tombolos prenant l'allure de cordons de galets. Pour autant, ils ne sont pas insolites car des constructions sédimentaires semblables ont été observées sur l'île de Keller au nord de Ouessant. D'autres formes d'accumulation de ce type, représentées par des empilements de dalles adossées à de petites falaises meubles, parsèment le littoral des îles de Molène et de Ouessant. Ces dépôts sont l'expression de processus d'accumulations supratidales de haute énergie qui attestent de la virulence des tempêtes océaniques du nord de l'Atlantique. Dans la perspective du réchauffement climatique, l'élévation du niveau marin associée à une augmentation de la fréquence des tempêtes ne pourrait qu'amplifier les processus hydrodynamiques et morphosédimentaires observés. À ce titre, cet objet morphologique apparaît comme un bon indicateur des variations météo-océaniques et/ou climatiques à plus ou moins long terme.

\section{Remerciements}

Nos remerciements vont à la DIREN Bretagne qui a financé ce travail, à J.-Y. Le Gall et D. Bourlès, gardes animateurs de la Réserve Naturelle d'Iroise, qui par tous temps nous ont permis d'accéder aux sites d'étude. Les données de houle issues de l'atlas numérique de mer océanique et côtier (ANEMOC), les enregistrements effectués par la bouéephare BEAIII et les données marégraphiques nous ont été fournis respectivement par Mme F. Lafon, MM. M. Benoit et J.-C. Fournier du LNHE-EDF, G. Goasguen du CETMEFBrest et B. Simon et R. Jehan du SHOM-Brest. Merci enfin à B. Hallégouët pour les photographies anciennes.

\section{Références}

Atwater B.F. (1992) - Geological evidence for earthquakes during the past 2000 years along the Copalis River, southern coastal 
Washington. Journal of Geophysical Research 97 (B2), 19011919.

Battjes J.A. (1974) - Surf similarity. Proceeding of the 14th Coastal Engineering Conference, American Society Civil Engineers 466-479.

Benoit M., Lafon F., Goasguen G. (2006) - Construction et exploitation d'une base de données d'états de mer le long des côtes françaises par simulation numérique sur 23 ans. In L'Her J., Levacher D. (Ed.) : Actes du colloque des IXe Journées Nationales Génie Civil - Génie Côtier, Brest, France, 12-14 septembre 2006, Centre Français du Littoral, Nantes, 21-30.

Betts N.L., Orford J.D., White D., Graham C.J. (2004) Storminess and surges in the south-western approaches of the eastern North Atlantic: the synoptic climatology of recent extreme coastal storms. Marine Geology 210, 227-246.

Bondevik S., Mangerud J., Dawson S., Dawson A., Lohne Ø. (2005) - Evidence for three North Sea tsunamis at the Shetland Islands between 8000 and 1500 years ago. Quaternary Science Reviews 24, 1757-1775.

Bouligand R., Pirazzoli P. A. (1999) - Les surcotes et les décotes à Brest, étude statistique et évolution. Oceanologica Acta 22, 2, 153-166.

Bourrouilh-Le Jan F. G., Talandier J. (1985) - Sédimentation et fracturation de haute énergie en milieu récifal : tsunamis, ouragans et cyclones et leurs effets sur la sédimentation et la géomorphologie d'un atoll : Motu et Hoa, à Rangiroa, Tuamotu, Pacifique SE. Marine Geology 67, 263-333.

Bryant E.A., Young R.W., Price D.M. (1992) - Evidence of tsunami sedimentation on the southeastern coast of Australia. The Journal of Geology 100, 753-765.

Caspar R., Costa S., Jakob E. (2007) - Fronts froids et submersions de tempête dans le nord-ouest de la France. Le cas des inondations par la mer entre l'estuaire de la Seine et la baie de Somme. La Météorologie, 57, 37-47.

Clague J.J., Hutchinson I., Mathewes R.W., Patterson R.T. (1999) - Evidence for late Holocene tsunamis at Catala Lake, British Columbia. Journal of Coastal Research 15, 1, 45-60.

Dawson A.G. (1994) - Geomorphological effects of tsunami runup and backwash. Geomorphology 10, 83-94.

Dawson A.G. (1999) - Linking tsunami deposits, submarine slides and offshore earthquakes. Quaternary International 60, 119-126.

Dawson A.G., Shi S. (2000) - Tsunami deposits. Pure and Applied Geophysics 157, 875-897.

Einsele G., Chough S.K., Shiki T. (1996) - Depositional events and their records - an introduction. Sedimentary Geology 104, 1-9.

Evans D.J.A., Archer S., Wilson D.J.H. (1999) - A comparison of the lichenometric and Schmidt hammer dating techniques based on data from the proglacial areas of some Islandic glaciers. Quaternary Science Reviews 18, 13-41.

Fichaut B., Hallégouët B. (1989) - Banneg : une île dans la tempête. Penn ar Bed, 135, 36-43.

Gianfreda F., Mastronuzzi G., Sansò P. (2001) - Impact of historical tsunamis on a sandy coastal barrier: an example from the northern Gargano cosat, southern Italy. Natural Hazards and Earth System Sciences 1, 213-219.
Goff J., Dudley W.C., de Maintenon M.J., Cain G., Coney J.P. (2006) - The largest local tsunami in 20th century Hawaii. Marine Geology 226, 65-79.

Guilcher A. (1959) - L'archipel de Molène (Finistère). Étude morphologique. Revue de Géographie Physique et de Géologie Dynamique, II, 2, 81-96.

Hallégouët B. (1982a) - Géomorphologie de l'archipel de Molène. Penn ar Bed, 110, 83-97.

Hallégouët B. (1982b) - L'archipel de Molène. Penn ar Bed, 110, 79-82.

Hallégouët B. (1984) - Contribution à l'étude morphologique de l'archipel de Molène (Finistère). Études géographiques sur la Bretagne et questions diverses. Actes du 107e Congrès National des Sociétés Savantes, Brest 1982, Secteur de Géographie, Paris, CTHS, 61-77.

Hallégouët B., Hénaff A. (2006) - Évolution récente et gestion des espaces littoraux de l'ouest Cornouaille. «Quelles pistes de développement pour le territoire? "Actes des rencontres de l'ouest Cornouaille, mai-juin 2005, Association Ouest Cornouaille Promotion, Pont-L'Abbé, 20-34.

Hartley A. Howell J., Mather A.E., Chong G. (2001) - A possible plio-pleistocene tsunami deposit, Hornitos, northern Chile. Revista Geológica de Chile 28, 1, 117-125.

Hearty P.J. (1997) - Boulder deposits from large waves during the last interglaciation on North Eleuthera Island, Bahamas. Quaternary Research 48, 326-338.

Jacob N., Gob F., Bravard J.-P., Petit F. (2006) - Les formes alluviales d'une rivière en gorge, le Chassezac (Cévennes, France). Géomorphologie : relief, processus, environnement, 1, 3-22.

Jones B., Hunter I.G. (1992) - Very large boulders on the coast of Grand Cayman: the effects of giant waves on rocky coastlines. Journal of Coastal Research 8, 4, 763-774.

Keating B., Whelan F., Bailey-Brock J. (2004) - Tsunami deposit at Queen's Beach, Oahu, Hawaii - Initial results and wave modelling. Science of Tsunami Hazards 22, 1, 23-43.

Kelletat D., Schellmann G. (2002) - Tsunamis on Cyprus: field evidences and ${ }^{14} \mathrm{C}$ dating results. Zeitschrift für Geomorphologie N. F. 46, 1, 19-34.

Liu K., Fearn M.L. (1993) - Lake-sediment record of late Holocene hurricane activities from coastal Alabama. Geology 21, 793-796.

Long D., Smith D.E., Dawson A.G. (1989) - A Holocene tsunami deposit in eastern Scotland. Journal of Quaternary Science 4, 1, 61-66.

Mase H. (1989) - Random wave runup height on gentle slopes. Journal of Waterway, Port, Coastal, and Ocean Engineering $115,5,649-661$.

Moore J.G., Moore G.W. (1984) - Deposit from a giant wave on the island of Lenai, Hawaii. Science 226, 1498-1499.

Morel V. (1997) - De la géomorphologie à la gestion des cordons de galets littoraux du bassin de la Manche et de ses abords : études de cas (Bretagne, Haute-Normandie, sud Angleterre) et réflexions générales, Thèse de géographie de l'université de Bretagne Occidentale, Brest, 386 p.

Nanayama F., Shigeno K., Satake K., Shimokawa S., Miyasaka S., Ishii M. (2000) - Sedimentary differences between the 
1993 Hokkaido-nansei-oki tsunami and the 1959 Miyakojima typhoon at Taisei, southwestern Hokkaido, northern Japan. Sedimentary Geology 135, 255-264.

Nataka T., Kawana T. (1993) - Historical and prehistorical large tsunamis in the southern Ryukus, Japan. Tsunami '93 Proceedings IUGG/IOC International Tsunami Symposium, Wakayama, Japan, August 23-27, 1993, 297-307.

Nichol S.L., Lian O.B., Carter C.H. (2003) - Sheet-gravel evidence for a late Holocene tsunami run-up on beach dunes, Great Barrier Island, New Zealand. Sedimentary Geology 155, 129-145.

Noormets R., Felton E.A., Crook K.A.W. (2002) - Sedimentology of rocky shorelines. 2. Shoreline clasts on the north of Oahu, Hawaii - origins and history. Sedimentary Geology 150, 31-45.

Noormets R., Crook K.A.W., Felton E.A. (2004) - Sedimentology of rocky shorelines. 3. Hydrodynamics of clast emplacement and transport on a shore platform, Oahu, Hawaii. Sedimentary Geology 172, 41-65.

Nott J. (1997) - Extremely high-energy wave deposit inside the Great Barrier Reef, Australia: determining the cause - tsunami or tropical cyclone. Marine Geology 141, 193-207.

Nott J. (2000) - Records of prehistoric tsunamis from boulder deposits - evidence from Australia. Science of Tsunami Hazards $18,1,3-14$.

Nott J. (2003) - Tsunami or storm waves? - determining the origin of a spectacular field of wave emplaced boulders using numerical storm surge and wave models and hydrodynamic transport equations. Journal of Coastal Research 19, 2, 348-356.

Nott J. (2004) - The tsunami hypothesis - comparisons of the field evidence against the effects, on the Western Australian coast, of some of the most powerful storms on Earth. Marine Geology 208, 1-12.

Nott J., Bryant E. (2003) - Extreme marine inundations (tsunamis?) of coastal western Australia. Journal of Geology 111, 691-706.
Paskoff R. (1991) - Likely occurrence of a mega-tsunami in the middle Pleistocene near Coquimbo, Chile. Revista Geológica de Chile 18, 87-91.

Pinegina T.K., Bourgeois J. (2001) - Historical and paleo-tsunami deposits on Kamchatka, Russia: long-term chronologies and long-distance correlations. Natural Hazards and Earth System Sciences 1, 177-185.

Pirazzoli P. A. (2000) - Surges, atmospheric pressure and wind change and flooding probability on the Atlantic coast of France. Oceanologica Acta, 23, 6, 643-661.

Pirazzoli P. A., Regnauld H., Lemasson L. (2004) - Changes in storminess and surges in western France during the last century. Marine Geology 210, 307-323.

Scheffers A., Kelletat D. (2004) - Bimodal tsunami deposits - a neglected feature in paleo-tsunami research. In Schernewski G., Dolch T. (Eds.): Geographie der Meere und Küsten, Coastline Reports 1, 67-75.

Whelan F., Keating B. (2004) - Tsunami deposits on the island of Oahu, Hawaiï. In Schernewski G., Dolch T. (Eds.): Geographie der Meere und Küsten, Coastline Reports 1, 77-82.

Winchester V., Chaujar R.K. (2002) - Lichenometric dating of slope movements, Nant Ffrancon, North Wales. Geomorphology 47, 61-74.

Winchester V., Harrison S. (2000) - Dendrochronology and lichenometry: colonization, growth rates and dating of geomorphological events on the east side of the north Patagonian icefield, Chile. Geomorphology 34, 181-194.

Young R.W., Bryant E.A., Price D.M. (1996) - Catastrophic wave (tsunami?) transport of boulders in southern New South Wales, Australia. Zeitschrift für Geomorphologie N.F, 40, 191-207.

Article soumis le 24 octobre 2007, accepté le 20 février 2008. 
